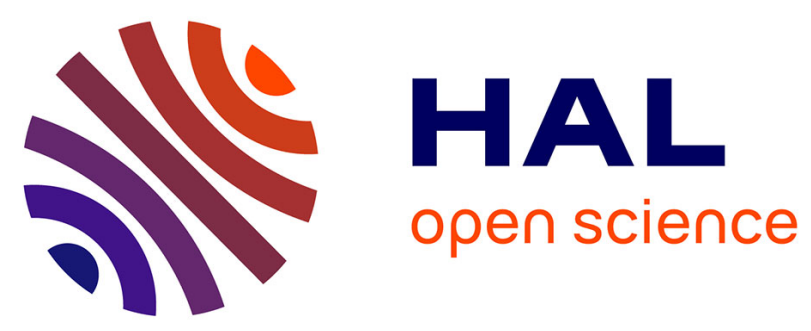

\title{
Copper- and Cobalt-Catalyzed Syntheses of Thiophene-Based Tertiary Amines
}

Salima Bouarfa, Simon Gra $\beta$ l, Maria Ivanova, Timothy Langlais, Ghenia

Bentabed-Ababsa, Frédéric Lassagne, William Erb, Thierry Roisnel, Vincent Dorcet, Paul Knochel, et al.

\section{To cite this version:}

Salima Bouarfa, Simon Gra $\beta$, Maria Ivanova, Timothy Langlais, Ghenia Bentabed-Ababsa, et al.. Copper- and Cobalt-Catalyzed Syntheses of Thiophene-Based Tertiary Amines. European Journal of Organic Chemistry, 2019, 2019 (20), pp.3244-3258. 10.1002/ejoc.201900276 . hal-02177982

\section{HAL Id: hal-02177982 https://hal-univ-rennes1.archives-ouvertes.fr/hal-02177982}

Submitted on 9 Jul 2019

HAL is a multi-disciplinary open access archive for the deposit and dissemination of scientific research documents, whether they are published or not. The documents may come from teaching and research institutions in France or abroad, or from public or private research centers.
L'archive ouverte pluridisciplinaire HAL, est destinée au dépôt et à la diffusion de documents scientifiques de niveau recherche, publiés ou non, émanant des établissements d'enseignement et de recherche français ou étrangers, des laboratoires publics ou privés. 


\title{
Copper- and Cobalt-Catalyzed Syntheses of Thiophene-Based
}

\section{Tertiary Amines}

\author{
Salima Bouarfa, ${ }^{[a, b]}$ Simon Graßl, ${ }^{[c]}$ Maria Ivanova, ${ }^{[c]}$ Timothy Langlais, ${ }^{[a, c]}$ Ghenia Bentabed-Ababsa, ${ }^{*[b]}$ \\ Frédéric Lassagne,${ }^{[\mathrm{a}]}$ William Erb, ${ }^{[\mathrm{a}]}$ Thierry Roisnel, ${ }^{[\mathrm{a}]}$ Vincent Dorcet, ${ }^{[\mathrm{a}]}$ Paul Knochel ${ }^{\star[\mathrm{c}]}$ and Florence \\ Mongin ${ }^{\star[a]}$
}

\begin{abstract}
Thienylzinc halides and related compounds prepared by deprotonation followed by transmetalation were used in coppercatalyzed amination using $\mathrm{N}$-benzoyloxy secondary amines. By extending the reaction to 1,5-naphthyridine, it was showed that the competitive dimer formation observed in the case of thiophenes was linked with the low stability of some thienylamines rather than homocoupling. Interestingly, thienylzinc halides and related compounds prepared by transmetalation of thienylmagnesium halides, either prepared from their bromo-precursors or generated by deprotometalation, were satisfactorily employed in cobalt-catalyzed aminations. Finally, aminothiophenes were involved in coppercatalyzed mono- and di- $\mathrm{N}$-arylations, affording differently substituted di- and triphenylamines.
\end{abstract}

\section{Introduction}

Electron-rich thiophenes and related compounds are an important class of five-membered aromatic heterocycles, notably for various applications in the fields of medicinal chemistry and materials. ${ }^{[1]}$ Thiophene for example is found in the skeleton of important pharmaceuticals such as duloxetine and olanzapine, which respectively target major depressive disorder and schizophrenia, and dorzolamide, an anti-glaucoma agent. The thiophene scaffold is also present in many organic materials among which we can cite light-emitting diodes, field effect transistors and solar cells. ${ }^{[1]}$ To access these aforementioned scaffolds, the functionalization of thiophenes is in general

[a] S. Bouarfa, T. Langlais, F. Lassagne, Dr. W. Erb, Dr. T. Roisnel, Dr. V. Dorcet, Prof. F. Mongin

Univ Rennes, CNRS, ISCR (Institut des Sciences Chimiques de Rennes) - UMR 6226

F-35000 Rennes, France

E-mail: florence.mongin@univ-rennes1.fr

https://iscr.univ-rennes1.fr/corint/florence-mongin

[b] S. Bouarfa, Prof. G. Bentabed-Ababsa

Laboratoire de Synthèse Organique Appliquée

Faculté des Sciences Exactes et Appliquées

Université Oran1 Ahmed Ben Bella, BP 1524 El M'Naouer

31000 Oran, Algeria

E-mail: badri_sofi@yahoo.fr

https://scholar.google.com/citations?user=7c5-Bw8AAAAJ\&hl=fr

[c] S. GraßI, Dr. M. Ivanova, T. Langlais, Prof. Dr. Paul Knochel Department Chemie

Ludwig-Maximilians-Universität München

Butenandtstrasse 5-13, Haus F, 81377 München, Germany

E-mail: paul.knochel@cup.uni-muenchen.de

http://www.knochel.cup.uni-muenchen.de/

Supporting information for this article is given via a link at the end of the document. preferred over cyclizations of suitable substrates. As a consequence, efficient methods to regio- and chemoselectively introduce substituents onto thiophenes and related compounds are required.

From the perspective of the pharmaceutical industry, one current challenge of organic synthesis remains the introduction of amines into drug molecules, if possible through $\mathrm{C}-\mathrm{H}$ bond activation, and more generally by formation of $\mathrm{C}-\mathrm{N}$ bonds. ${ }^{\left[{ }^{2]}\right.}$ Heteroaromatic $\mathrm{C}\left(\mathrm{sp}^{2}\right)-\mathrm{N}$ bonds can be formed by transition metal-catalyzed couplings from the corresponding halides, ${ }^{[3]}$ either using palladium- ${ }^{[4]}$ or copper-catalysts. ${ }^{[5]}$ Two complementary approaches have since been developed for the direct functionalization of heteroarenes, ${ }^{[6]}$ oxidative reactions in the presence of a suitable metal catalyst-oxidant combination, ${ }^{[7]}$ and transition metal-catalyzed electrophilic amination reactions. ${ }^{[8]}$

Among the methods used to introduce aliphatic amines onto thiophenes, the oxidative nucleophilic substitution of hydrogen has been described but remains limited to specific nitro compounds $;{ }^{[9]}$ therefore, most of the studies follow one of the above-mentioned strategies. Zinc amidocuprates formed from thienylzincs and lithium amides were involved in copper(I)mediated oxidative aminations. ${ }^{[10]}$ Owing to a picolinamide directing group, morpholine can be introduced onto thiophene in the presence of a copper(II)-catalyst and $\mathrm{Phl}(\mathrm{OAc})_{2}$ as oxidant. ${ }^{[11]}$ The in situ formation of diaryl- $\lambda 3$-iodanes followed by copper(I)-catalyzed reaction with morpholine can be similarly applied to thiophene in average yields. ${ }^{[12]}$ Provided that a carboxamide group is present, ruthenium(II)-catalyzed $\mathrm{C}-\mathrm{H}$ amination of thiophenes can be performed with $\mathrm{N}$ (benzoyloxy)morpholine in moderate yields. ${ }^{[13]}$ The use of $O$ benzoyl hydroxylamines $\left(\mathrm{BzO}-\mathrm{NR}_{2}\right)$ to intercept thienylmetals under copper(I)-catalysis displays a broader substrate scope, and has been developed from arylmetals prepared by $\mathrm{C}-\mathrm{H}$ lithiation-transalumination ${ }^{[14]}$ and, above all, by deprotozincation. ${ }^{[15]}$

If copper was employed as catalyst in the Johnson pioneered electrophilic amination of diarylzincs by $\mathrm{BzO}-\mathrm{NR}_{2}$ in $2004,{ }^{[16]}$ cobalt can also be used to catalyze amination of arylzincs. ${ }^{[8 b]}$ Indeed, we recently showed that both arylzinc pivalates $^{[17]}$ and chlorides ${ }^{[18]}$ can be involved in such an amination in the presence of cobalt salts. In the present paper, we report our studies on copper- and cobalt-catalyzed reactions involving thienylzincs and $O$-benzoyl secondary hydroxylamines. Our investigations on the copper-catalyzed mono and double $\mathrm{N}$ arylation of thienylamines by aromatic iodides to access thiophene-based triarylamines are also presented. 


\section{Results and Discussion}

We first studied a 'deprotolithiation-transmetalation to zinccopper catalyzed amination' sequence in THF (THF = tetrahydrofuran) by starting from benzothiophene (Table 1). In order to generate the corresponding arylzinc halide, we compared two methods, (i) butyllithium-mediated deprotolithiation followed by transmetalation using $\mathrm{ZnCl}_{2} \cdot \mathrm{TMEDA}^{[19]} \quad\left(\right.$ TMEDA $\quad=\quad N, N, N^{\prime}, N^{\prime}-$ tetramethylethylenediamine; entry 1) and (ii) deprotolithiation using LiTMP (TMP = 2,2,6,6-tetramethylpiperidino) in the presence of $\mathrm{ZnCl}_{2}$.TMEDA as in situ trap (entry 2). ${ }^{[20]}$ Amination was next performed by using $\mathrm{N}$-(benzoyloxy)diallylamine and a catalytic amount of copper(II) acetate. Under these conditions, the expected product 1a was isolated in $51-52 \%$ yield in spite of its propensity to turn into 2,2'-bi(benzothiophene) (1'; entries 1 and 2). These results suggest that lithium chloride and TMEDA, that are both present, do not hamper the reaction. In contrast, attempts to directly use benzothienyllithium (formed by reaction with LiTMP) instead of the corresponding zinc compound did not furnish 1a (entry 3). The presence of the electrophile and catalyst during the addition of the base to the substrate similarly failed (entry 4). N-(benzoyloxy)dibutylamine, $\mathrm{N}$ (benzoyloxy)morpholine and $\mathrm{N}$-(benzoyloxy)piperidine were used under the best reaction conditions to afford the expected benzothienylamines 1b-d (entries 5-7; Figure 1, top).

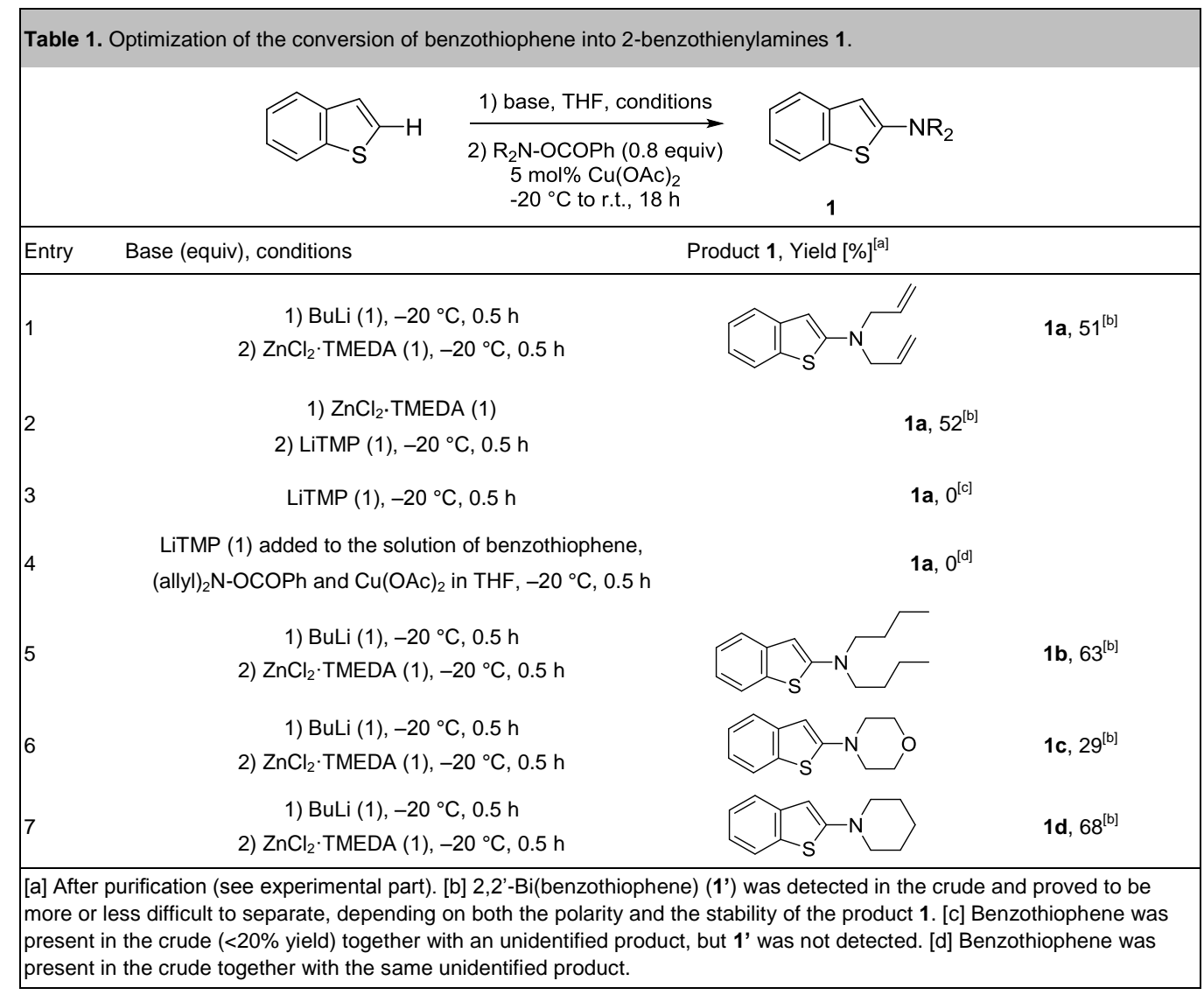

1) BuLi (1 equiv), THF, $-20^{\circ} \mathrm{C}, 0.5 \mathrm{~h}$ 2) $\mathrm{ZnCl}_{2} \cdot \operatorname{TMEDA}$ (1 equiv), $-20^{\circ} \mathrm{C}, 0.5 \mathrm{~h}$<smiles>Clc1cccs1</smiles>

2) $\mathrm{R}_{2} \mathrm{~N}-\mathrm{OCOPh}(0.8$ equiv) $5 \mathrm{~mol} \% \mathrm{Cu}(\mathrm{OAc})_{2},-20^{\circ} \mathrm{C}$ to $\mathrm{rt}, 18 \mathrm{~h}$<smiles>C=CCN(CC=C)c1ccc(Cl)s1</smiles>

$(49 \% \text { yield })^{[a]}$

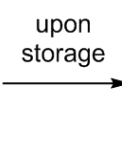

upon<smiles>Clc1ccc(-c2ccc(Cl)s2)s1</smiles>

$2 a^{\prime}$<smiles>C=CC[N+](CC=C)c1ccc(Cl)s1</smiles>

Scheme 1. Extension of the sequence to 2 -chlorothiophene and proposed mechanism to rationalize the degradation of $2 \mathbf{a}$. [a] 5,5'Dichloro-2,2'-bithiophene (2a') was also isolated in $32 \%$ yield. 
When the reaction was extended to 2-chlorothiophene, we isolated both the expected diallylamine $2 \mathbf{a}$ in $49 \%$ yield and the dimer $\mathbf{2 a}$ ' in $32 \%$ yield (Scheme 1). Interestingly we observed that, upon storage in a fridge, isolated $2 \mathbf{a}$ turns dark blue in a few days (or in a few minutes if dissolved in chloroform) while it is converted into the dimer 2a'. This result tends to show that dimerization is not related to a side homocoupling taking place in the course of the reaction, but rather associated with the thienylamine instability. ${ }^{[21]}$ We thus decided to move to a substrate for which the corresponding amines are stable.

The 1,5-naphthyridine heterocycle is present in numerous compounds endowed with biological properties as well as in OLED materials. ${ }^{[22]}$ Besides direct introduction of an amino group by a Chichibabin reaction, ${ }^{[23]}$ its functionalization at C4 can be achieved by deprotometalation. The latter was performed by using a lithium amide provided that a directing group is present at $\mathrm{C} 3{ }^{[24]}$ However, from bare 1,5-naphthyridine, a regioselective reaction at $\mathrm{C} 4$ was found possible after precomplexation of the substrate with $(\mathrm{TMP})_{2} \mathrm{Mg} \cdot 2 \mathrm{LiCl}$, and takes place in THF within $5 \mathrm{~min}$ at $-78^{\circ} \mathrm{C} .^{[22]}$ In order to prepare amines (Table 2), we first evaluated the 'deprotolithiationtransmetalation to zinc-copper catalyzed amination' sequence, successful from benzothiophene, by using $\mathrm{N}$ (benzoyloxy)diallylamine as electrophile. However, under these conditions, the expected amine $\mathbf{3 a}$ was isolated in a maximum $10 \%$ yield (entries 1 and 2 ). In order to understand the reason of this failure, we tested iodine as electrophile, and obtained the 4iodo 3-I and 4,8-diiodo 3'-I in respective yields of 34 and 6\% alongside with remaining starting material (Scheme 2, left). Concerned by the potential low stability of the intermediate 4lithio compound, we next moved to more stable zinc amides. After deprotozincation using TMPZnCl. $\mathrm{LiCl}^{[25]}$ (1.1 equiv) at room temperature for $2 \mathrm{~h}$, interception with $\mathrm{N}$ (benzoyloxy)diallylamine as before led to $3 a$ in $64 \%$ yield (entry 3 ), a rather good result since trapping with iodine afforded $3-1$ in $72 \%$ yield (Scheme 2, left). (TMP) ${ }_{2} \mathrm{Zn} \cdot 2 \mathrm{LiCl}$, prepared from LiTMP and $\mathrm{ZnCl}_{2}$ in a 2:1 ratio, was similarly employed and furnished the amine $3 \mathrm{c}$ after copper-catalyzed reaction with $\mathrm{N}$ (benzoyloxy)morpholine (entry 4). Interestingly, a magnesium intermediate generated by TMPMgCl. $\mathrm{LiCl}{ }^{[26]}$ is tolerated, ${ }^{[27]}$ as evidenced by quenching with $\mathrm{N}$-(benzoyloxy)diisopropylamine to afford the amine $3 \mathbf{b}$ in $52 \%$ yield (entry 5 ).

Alternatively, the synthesis of the amines 3a-c can be considered by nucleophilic substitution of the iodo 3-I. If such a possibility efficiently worked by using morpholine as nucleophile, the yield dropped to $17 \%$ with diallylamine, and the reaction failed with diisopropylamine, a result probably due to important steric hindrance in this case (Scheme 2, right). Thus, deprotometalation-amination and deprotometalation-iodolysissubstitution appear as being complementary to access 1,5naphthyridines 4-substituted by various amino groups.

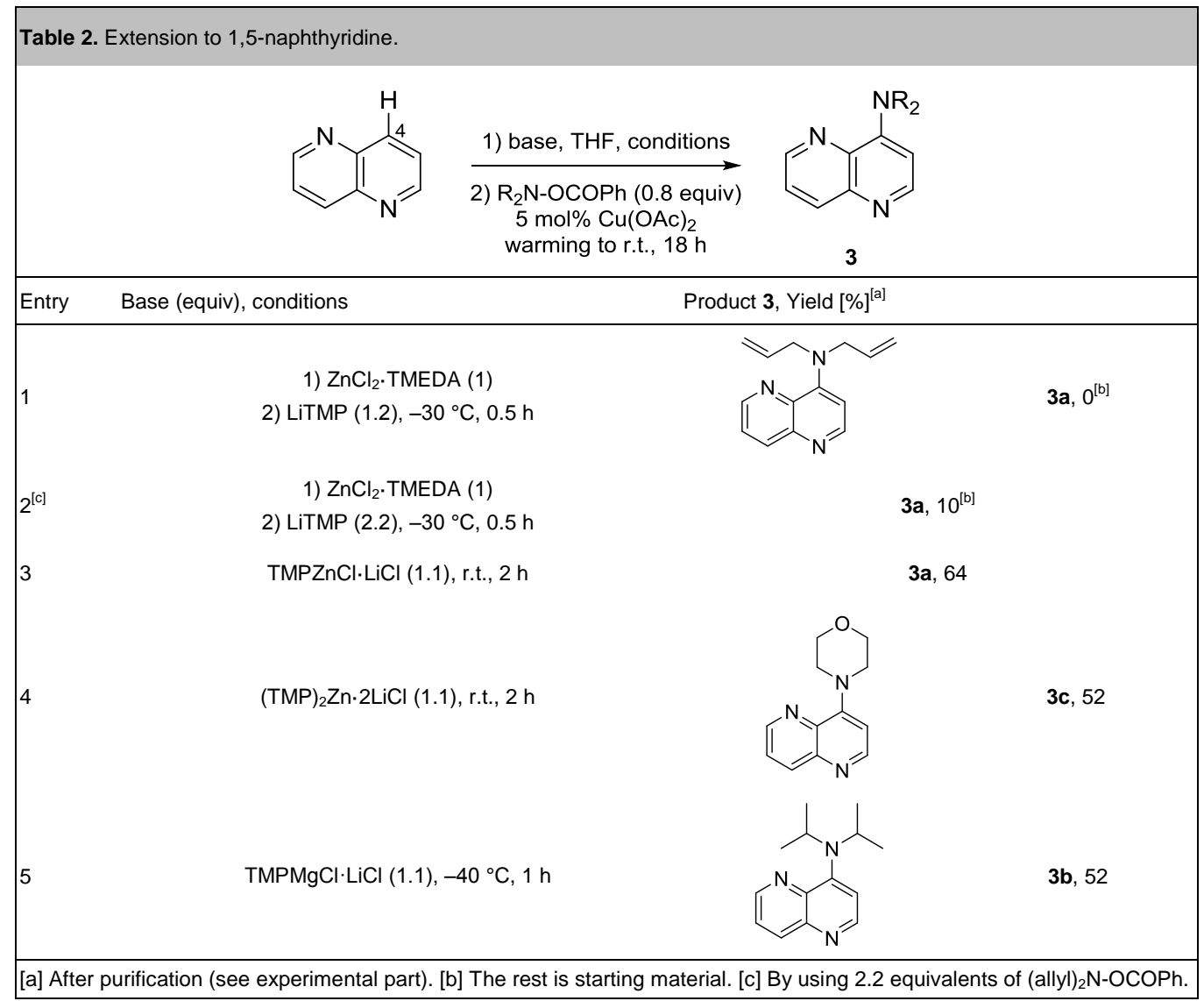


<smiles></smiles>

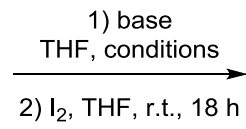<smiles>Ic1ccnc2cccnc12</smiles><smiles>C=CCN(CC=C)c1ccnc2cccnc12</smiles>

3a, $17 \%$<smiles>CC(C)N(c1ccnc2cccnc12)C(C)C</smiles>

3b, traces<smiles>c1cnc2c(N3CCOCC3)ccnc2c1</smiles>

3c, $70 \%$

$\mathrm{ZnCl}_{2} \cdot$ TMEDA ( 1 equiv) then LiTMP

( 1.5 equiv), $-30{ }^{\circ} \mathrm{C}, 0.5 \mathrm{~h}: 34 \%[\mathrm{a}]$

TMPZnCl. LiCl ( 1.2 equiv), r.t., 2 h: $72 \%$

Scheme 2. Alternative way to access 1,5-naphthyridines 4-substituted by amino groups. [a] 4,8-Diiodo-1,5-naphthyridine (3'-I) was also isolated in $6 \%$ yield.

We next involved thiophene-based and related organozinc chlorides in cobalt-catalyzed amination reactions. First, arylzinc compounds were prepared by transmetalation of the corresponding arylmagnesium reagents obtained by $\mathrm{LiCl}$ favored reactions: 2-thienyl- and 2-benzothienylmagnesium bromides by Grignard reaction (insertion method $\mathbf{A} ;{ }^{[28]}$ Scheme 3, top), and 2-thiazolyl- and 2-benzothiazolylmagnesium bromides by bromine/metal exchange (exchange method $\mathbf{B}^{[29]}$ bottom). The amination step was successfully performed to respectively afford $\mathbf{1 c}, \mathbf{2 c}, \mathbf{2 e}, \mathbf{2 f}$ and $\mathbf{4 c}, 5 \mathbf{e}, \mathbf{5 f}, \mathbf{5 g}$ by using $\mathrm{N}$ (benzoyloxy) secondary amines in the presence of $\mathrm{CoCl}_{2} \cdot 2 \mathrm{LiCl},{ }^{[17]}$ a combination that recently proved efficient to functionalize organozincs prepared from other aryl halides. ${ }^{[17-18]}$

For atom economy reason and to avoid the use of costly aryl bromides, we finally employed organozinc compounds prepared by deprotometalation-transmetalation in this cobalt-catalyzed amination (Scheme 4). This represents an additional challenge as very few cobalt-catalyzed electrophilic trappings have been achieved from arylzincs prepared by deprotometalation. ${ }^{[30]}$

To this purpose, we involved in the amination 2-thienylzinc chlorides prepared from 2,2'-bithiophene and thieno[3,2b]thiophene by deprotomagnesiation using $\mathrm{TMPMgCl} \cdot \mathrm{LiCl}^{[26,31]}$ followed by transmetalation (top). Similarly, we performed the amination of the organozinc chloride coming from 1,3-dithiole-2thione, generated by using the same base at low temperature ${ }^{[32]}$ followed by transmetalation (bottom). The reactions gave the expected amines $\mathbf{6 c}, \mathbf{7 c}, \mathbf{7 f}, \mathbf{7 h}$ and $\mathbf{8 c}, \mathbf{8 e}, \mathbf{8 f}, \mathbf{8 h}$ in high yields in the presence of TMEDA ( 0.2 equiv), ${ }^{[17]}$ making this cobaltcatalyzed 'deprotonation-transmetalation-amination' a general way to obtain amino-substituted thiophenes and related compounds.

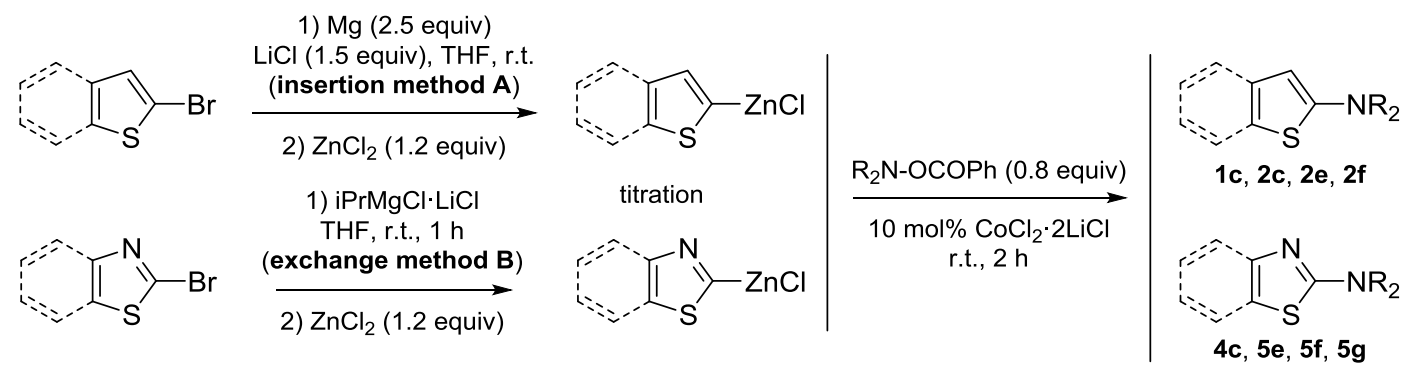<smiles>c1ccc2sc(N3CCOCC3)cc2c1</smiles>

1c: $96 \%{ }^{[a]}$<smiles>c1csc(N2CCOCC2)n1</smiles>

4c: $76 \%[\mathrm{a}]$<smiles>c1csc(N2CCOCC2)c1</smiles>

2c: $80 \%[a]$<smiles>COCCN(CCOC)c1cccs1</smiles>

2e: $52 \%[a]$<smiles>c1ccc2sc(N3CCC4(CC3)OCCO4)nc2c1</smiles>

5f: $88 \%[a]$<smiles>c1csc(N2CCC3(CC2)OCCO3)c1</smiles>

2f: $78 \%{ }^{[a]}$<smiles>COCCN(CCOC)c1nc2ccccc2s1</smiles>

5e: $56 \%[a]$<smiles>c1ccc2sc(N3CCCCCC3)nc2c1</smiles>

5g: $89 \%[a]$

Scheme 3. Cobalt-catalyzed amination of (benzo)thienylzinc and (benzo)thiazolylzinc chlorides prepared from the corresponding halides. ${ }^{[a]}$ Yields are given after purification (see experimental part). 


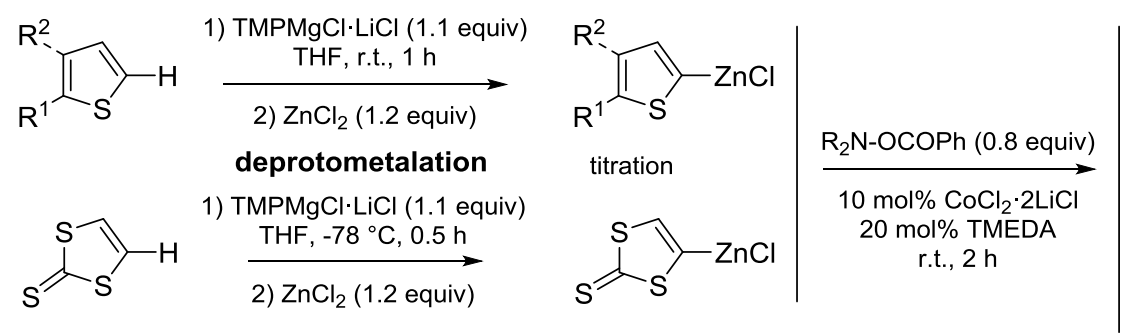

$\mathrm{R}_{\mathbf{6 c}, 7 \mathrm{c}, 7 \mathrm{f}, 7 \mathrm{~h}}^{\mathrm{R}^{2}}$<smiles></smiles><smiles>c1csc(-c2ccc(N3CCOCC3)s2)c1</smiles><smiles>S=c1scc(N2CCOCC2)s1</smiles>

8c: $73 \%{ }^{[a]}$<smiles>c1cc2sc(N3CCOCC3)cc2s1</smiles>

7c: $92 \%{ }^{[a]}$<smiles>COCCN(CCOC)c1csc(=S)s1</smiles>

8 e: $95 \%[a]$<smiles>c1cc2sc(N3CCC4(CC3)OCCO4)cc2s1</smiles>

7f: $85 \%$ [a]<smiles>S=c1scc(N2CCC3(CC2)OCCO3)s1</smiles>

8f: $98 \%[$ [a]<smiles>c1ccc2c(c1)CCN(c1cc3sccc3s1)C2</smiles>

7h: $88 \%^{[a]}$<smiles>S=c1scc(N2CCc3ccccc3C2)s1</smiles>

8h: $96 \%^{[a]}$

Scheme 4. Cobalt-catalyzed amination of organozinc chlorides prepared by deprotonation-transmetalation. ${ }^{\text {[a] }}$ Yields are given after purification (see experimental part).

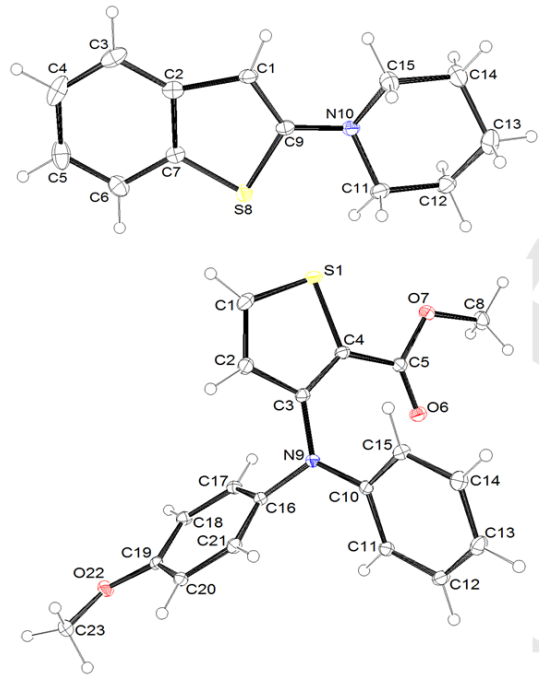

Figure 1. ORTEP diagrams (50\% probability) of the compounds $1 \mathrm{~d}$ and $10 \mathrm{ab}$.

Apart from nucleophilic substitution reactions that require activated aryl halides and strong reaction conditions, ${ }^{[33]}$ access to thiophene-based triarylamines is more readily possible by applying the Buchwald-Hartwig approach. Thus, palladiumcatalyzed reactions have been used to $N$-arylate 2 -amino- ${ }^{[33 a, 34]}$ and 3 -amino- ${ }^{[35]}$ thiophenes or benzothiophenes. To avoid the use of expensive transition metal, and following our interest in $\mathrm{N}$ arylation reactions, ${ }^{[36]}$ we next investigated the copper-catalyzed $\mathrm{N}$-arylation of thiophene-based anilines. Indeed, if copper has been employed to catalyze $\mathrm{N}$-arylation of an aminothiophene with organoboron reagents, ${ }^{[37]}$ aryl halides have to our knowledge never been used in such reactions.
We recently reported the use of 2-aminophenones as substrates in copper-catalyzed $\mathrm{N}$-arylation with aryl and heteroaryl iodides. ${ }^{[36 \mathrm{~d}]}$ To achieve the reactions, we employed a catalytic amount of activated copper and potassium carbonate as a base at the reflux temperature of dibutylether. We chose these conditions as starting point to investigate the reaction from methyl 3-amino-2-thiophenecarboxylate and methyl 2-amino-3thiophenecarboxylate. Preliminary results led us to rapidly realize that reproducibility of the reactions could be ensured by using stoichiometric copper ( $5 \times 0.2$ equiv added every $2 \mathrm{~h}$ ), conditions that were kept to perform the whole study.

When methyl 3-amino-2-thiophenecarboxylate (Scheme 5) was treated by 1.5 equivalents of iodobenzene, a mixture of the mono- and diphenylated products $9 \mathrm{a}$ and $10 \mathrm{a}$ was formed in a $\sim 1: 1$ ratio under these conditions. By reducing the amount of aryl iodide to one equivalent, monoarylation was favored, affording 9 a in $79 \%$ yield; only traces of $\mathbf{1 0 a}$ were observed in the crude, and could be easily removed by purification over silica gel (top). Conversely, increasing the amount of iodide to 2 equivalents allowed 10a to be obtained in a high $88 \%$ yield; using 3iodothiophene instead of iodobenzene similarly furnished $10 \mathrm{~d}$ (bottom left).

The formation of the monophenylated $9 \mathrm{a}$ in high yield using 1 equivalent of iodide shows that $9 \mathbf{a}$ is less reactive than starting 3-amino-2-thiophenecarboxylate in the $\mathrm{N}$-arylation reaction. It is nevertheless possible, by using an excess of the iodide, to prepare triarylamines, as shown with the products $10 \mathrm{a}$ and $10 \mathrm{~d}$. Furthermore, a different aryl iodide could be used in the second $\mathrm{N}$-arylation reaction and, when 9 a was treated by 4 -iodoanisole and 1-iodo-4-(trifluoromethyl)benzene under the same reaction conditions, the differently substituted triarylamines $10 \mathrm{ab}$ (Figure 1 , bottom) and $10 \mathrm{ac}$ were isolated in high yields (bottom right). 
<smiles>COC(=O)c1sccc1N</smiles>

$$
\begin{gathered}
\text { I-Ph (1 equiv) } \\
\begin{array}{c}
\text { activated Cu (1 equiv) } \\
\mathrm{K}_{2} \mathrm{CO}_{3} \text { (2 equiv) }
\end{array} \\
\underset{\mathrm{Bu}}{\mathrm{Bu}_{2} \mathrm{O}, 140{ }^{\circ} \mathrm{C}, 24 \mathrm{~h}} \\
(79 \%)
\end{gathered}
$$

I-Ar (2 equiv) activated $\mathrm{Cu}$ (1 equiv) ${ }^{[\mathrm{a}]}$ $\mathrm{K}_{2} \mathrm{CO}_{3}$ (2 equiv) $\mathrm{Bu}_{2} \mathrm{O}, 140^{\circ} \mathrm{C}, 24 \mathrm{~h}$<smiles>COC(=O)c1sccc1N(c1ccccc1)c1ccccc1</smiles>

10a: $88 \%{ }^{[b]}$<smiles>COC(=O)c1sccc1N(c1ccsc1)c1ccsc1</smiles>

10d: $87 \%[$ [b]<smiles>COC(=O)c1sccc1N(c1ccccc1)c1ccc(OC)cc1</smiles>

10ab: $91 \%^{[b]}$<smiles>COC(=O)c1sccc1Nc1ccccc1</smiles>

I-Ar (1 equiv) activated $\mathrm{Cu}$ (1 equiv) ${ }^{[a]}$ $\mathrm{K}_{2} \mathrm{CO}_{3}$ (2 equiv) $\mathrm{Bu}_{2} \mathrm{O}, 140^{\circ} \mathrm{C}, 24 \mathrm{~h}$

Scheme 5. $\mathrm{N}$-arylation of methyl 3-amino-2-thiophenecarboxylate to afford either $\mathbf{9}$ or $\mathbf{1 0}$. [a] Added by portions of 0.2 equivalent every 2 h. [b] After purification (see experimental part).<smiles>COc1ccc(I)cc1</smiles>

(1 equiv)<smiles>FC(F)(F)c1ccc(I)cc1</smiles>

(1 equiv)<smiles>COC(=O)c1sccc1Nc1ccccc1</smiles>

activated $\mathrm{Cu}$ ( 1 equiv) $\mathrm{K}_{2} \mathrm{CO}_{3}$ (2 equiv) $\mathrm{Bu}_{2} \mathrm{O}, 140{ }^{\circ} \mathrm{C}, 24 \mathrm{~h}$<smiles>COC(=O)c1sccc1N(c1ccccc1)c1ccc(OC)cc1</smiles><smiles>COC(=O)c1sccc1N(c1ccccc1)c1ccc(C(F)(F)F)cc1</smiles>

10ab: $42 \%$ yield $10 \mathrm{ac}: 28 \%$ yield

Scheme 6. Compared reactivities of 4-iodoanisole and 1-iodo-4-(trifluoromethyl)benzene in the $N$-arylation of 9 a.

A competitive reaction performed on $9 \mathbf{a}$ in the presence of two halides, 4-iodoanisole and 1-iodo-4-(trifluoromethyl)benzene showed close reactivities, but in favor of more electron-rich 4iodoanisole (Scheme 6). When compared with works on both the Goldberg reaction ${ }^{[38]}$ and the copper-catalyzed $N$-arylation of aniline with aryl bromides, ${ }^{[39]}$ in which electron-withdrawing substituents at the para position of the aryl halide facilitate the reaction rates, our competition study revealed a different behavior for the 4-trifluoromethylated iodide.

We next turned to the isomeric methyl 2-amino-3thiophenecarboxylate (Scheme 7). As observed for methyl 3amino-2-thiophenecarboxylate, reaction with 1.5 equivalents of iodobenzene under the same reaction conditions led to a mixture of the mono- and diphenylated products 11a and 12a in respective yields of $59 \%$ and $30 \%$. It proved easy to achieve monophenylation by using 1 equivalent of iodobenzene $(83 \%$ yield; top). Though somewhat less efficient, diphenylation was also possible $(54 \%$ and $76 \%$ yield, respectively using iodobenzene and 3-iodothiophene; bottom left). The $\mathrm{N}$-arylation of 11a with 4-iodoanisole and 2-iodobenzofuran satisfactorily led to two new thiophene-based triarylamines (bottom right).

We finally compared the reactivity of 2-aminothiophenes and 3 -aminothiophenes in this $\mathrm{N}$-arylation reaction (Scheme 8). To this purpose, we chose 4-iodoanisole to first attempt a competitive reaction between methyl 3-amino-2thiophenecarboxylate and methyl 2-amino-3thiophenecarboxylate (left). Even if the former also reacted, we noticed a higher reactivity of the latter (a $\sim 1: 3$ ratio was recorded). As monophenylated $9 \mathrm{a}$ and $11 \mathrm{a}$ are less reactive than their precursors (at the origin of the possible monoarylation reactions), this reactivity difference towards 4-iodoanisole is logically more pronounced between less reactive $9 \mathrm{a}$ and $11 \mathrm{a}$ (right). It is difficult to rationalize such a reactivity difference, but more studies will be performed in order to get general trends and connect them with possible reaction mechanisms. 
<smiles>COC(=O)c1ccsc1N</smiles>

-Ar (2 equiv) activated $\mathrm{Cu}\left(1\right.$ equiv) ${ }^{[a]}$ $\mathrm{K}_{2} \mathrm{CO}_{3}$ (2 equiv) $\mathrm{Bu}_{2} \mathrm{O}, 140^{\circ} \mathrm{C}, 24 \mathrm{~h}$<smiles>COC(=O)c1ccsc1N(c1ccccc1)c1ccccc1</smiles>

12a: $54 \%[c]$<smiles>COC(=O)c1ccsc1N(c1ccsc1)c1ccsc1</smiles>

12d: $76 \%$ [c]
I-Ph (1 equiv) activated $\mathrm{Cu}$ (1 equiv) ${ }^{[\mathrm{a}]}$ $\mathrm{K}_{2} \mathrm{CO}_{3}$ (2 equiv)

$\mathrm{Bu}_{2} \mathrm{O}, 140^{\circ} \mathrm{C}, 24 \mathrm{~h}$ $(83 \%)^{[b]}$<smiles>COC(=O)c1ccsc1Nc1ccccc1</smiles>

11a
I-Ar (1 equiv) activated $\mathrm{Cu}$ (1 equiv) ${ }^{[\mathrm{a}]}$ $\mathrm{K}_{2} \mathrm{CO}_{3}$ (2 equiv) $\mathrm{Bu}_{2} \mathrm{O}, 140^{\circ} \mathrm{C}, 24 \mathrm{~h}$ 12d: $76 \%$<smiles>COC(=O)c1ccsc1N(c1ccccc1)c1ccc(OC)cc1</smiles>

12ab: $86 \%[c]$

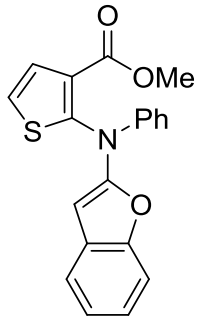

12ae: $53 \%{ }^{[c, d]}$
Scheme 7. $\mathrm{N}$-arylation of methyl 2-amino-3-thiophenecarboxylate to afford either 11 or 12 . [a] Added by portions of 0.2 equivalent every $2 \mathrm{~h}$. [b] The diphenylated product 12a was also isolated in 6\% yield. [c] After purification (see experimental part). [d] Starting iodide and benzofuran were present in the crude, but no more aminothiophene.<smiles>COC(=O)c1sccc1N</smiles>

(1 equiv)<smiles>COC(=O)c1sccc1Nc1ccc(OC)cc1</smiles>

9b:11b ratio: $26 / 74$<smiles>COC(=O)c1ccsc1N</smiles>

(1 equiv)<smiles>COc1ccc(I)cc1</smiles><smiles>COC(=O)c1ccsc1Nc1ccc(OC)cc1</smiles><smiles>COC(=O)c1sccc1Nc1ccccc1</smiles>

9a (1 equiv)

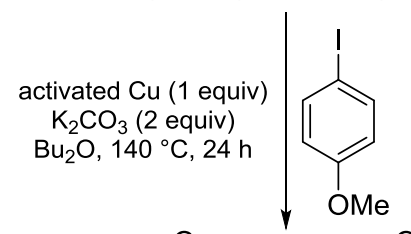<smiles>COC(=O)c1sccc1N(c1ccccc1)c1ccc(OC)cc1</smiles>

10ab: not formed<smiles>COC(=O)c1ccsc1Nc1ccccc1</smiles>

11a (1 equiv)<smiles>COC(=O)c1ccsc1N(c1ccccc1)c1ccc(OC)cc1</smiles>

12ab: only product formed

Scheme 8. Left: Compared reactivities of methyl 3-amino-2-thiophenecarboxylate and methyl 2-amino-3-thiophenecarboxylate in the $\mathrm{N}$-arylation using 4-iodoanisole. Right: Compared reactivities of methyl 3-(phenylamino)-2-thiophenecarboxylate (9a) and methyl 2(phenylamino)-3-thiophenecarboxylate (11a) in the $\mathrm{N}$-arylation using 4-iodoanisole. 


\section{Conclusions}

In the course of this study dedicated to the synthesis of thiophene-based tertiary amines, we have developed three approaches that are copper-catalyzed and cobalt-catalyzed amination of thienylzinc halides or related compounds, and copper-catalyzed arylation of aminothiophenes. The amination allowed thienylzinc halides, obtained by bromine/metal exchange but also by deprotometalation, to be functionalized; the only limit seems to be the low stability of some of the generated thienylamines. Concerning copper-catalyzed $\mathrm{N}$ arylation of aminothiophenes, a single reaction can be selectively achieved, but also diarylation using two different aryl iodides. Such reactions will undoubtly find applications given the interest for thiophenes and related compounds in the fields of medicinal chemistry and materials.

\section{Experimental Section}

\section{General}

All reactions were carried out under an argon atmosphere in flame-dried glassware. Syringes, which were used to transfer anhydrous solvents or reagents, were purged with argon prior to use. THF was continuously refluxed and freshly distilled from sodium-benzophenone under nitrogen or argon and stored over molecular sieves. Column chromatography separations were achieved on silica gel (40-63 $\mu \mathrm{m})$ from Merck. Melting points were measured on a Kofler apparatus. IR spectra were taken on a Perkin-Elmer Spectrum 100 spectrometer. ${ }^{1} \mathrm{H}$ and ${ }^{13} \mathrm{C}$ Nuclear Magnetic Resonance (NMR) spectra were recorded either on a Bruker Avance III spectrometer at $300 \mathrm{MHz}$ and $75 \mathrm{MHz}$ respectively, or on a Bruker ARX400 spectrometer at $400 \mathrm{MHz}$ and $101 \mathrm{MHz}$ respectively. ${ }^{1} \mathrm{H}$ chemical shifts $(\delta)$ are given in parts per million (ppm) relative to the solvent residual peak and ${ }^{13} \mathrm{C}$ chemical shifts are relative to the central peak of the solvent signal. ${ }^{[40]}$ Mass spectra and high resolution mass spectra (HRMS) were recorded using electron ionization (EI) except otherwise noted. Gas-chromatographical analyses were performed on machines of the types Hewlett-Packard 6890 or 5890 Series II (Hewlett Packard, 5\% phenylmethylpolysiloxane; length: $10 \mathrm{~m}$, diameter: $0.25 \mathrm{~mm}$; film thickness: $0.25 \mu \mathrm{m}$ )

\section{Starting materials}

iPrMgCl. $\mathrm{LiCl}$ was purchased as a solution in THF from Albemarle and titrated against iodine prior to use. ${ }^{[41]}$

$\mathrm{ZnCl}_{2}$ solution (1.0 M in THF) was prepared by drying $\mathrm{ZnCl}_{2}(136 \mathrm{~g}$, $0.10 \mathrm{~mol}$ ) in a Schlenk-flask under vacuum at $140^{\circ} \mathrm{C}$ for $5 \mathrm{~h}$. After cooling, dry THF (100 mL) was added and stirring was continued until all salts were dissolved $(12 \mathrm{~h}) . \mathrm{ZnCl}_{2} \cdot \mathrm{TMEDA}$ was prepared as reported previously. ${ }^{[19 a]}$

After opening a new bottle of 2,2,6,6-tetramethylpiperidine, $\mathrm{KOH}$ pellets were added and storage in a desiccator was necessary. LiTMP was prepared by adding BuLi (about $1.6 \mathrm{M}$ hexanes solution, $1.5 \mathrm{mmol}$ ) to a stirred, cooled $\left(-10^{\circ} \mathrm{C}\right)$ solution of $2,2,6,6$-tetramethylpiperidine $(0.28 \mathrm{~mL}$
$1.7 \mathrm{mmol})$ in THF ( $3 \mathrm{~mL}$ ) and stirring for $5 \mathrm{~min}$. If necessary, the solution was titrated as reported previously. ${ }^{[26]}$

TMPZnCl.LiCl was prepared as follows. ${ }^{[25]}$ A dry and argon flushed $250 \mathrm{~mL}$ Schlenk flask, equipped with a magnetic stirrer and a septum, was charged with 2,2,6,6-tetramethylpiperidine $(10.2 \mathrm{~mL}, 60 \mathrm{mmol})$ and THF $(60 \mathrm{~mL})$. The solution was cooled to $-40^{\circ} \mathrm{C}$ before dropwise addition of BuLi (2.4 M in hexane, $25 \mathrm{~mL}, 60 \mathrm{mmol})$. Then, the reaction mixture was allowed to warm up slowly to $-10^{\circ} \mathrm{C}$ for $1 \mathrm{~h} . \mathrm{ZnCl}_{2}(1.0 \mathrm{M}$ in THF, $66 \mathrm{~mL}, 66 \mathrm{mmol}$ ) was added dropwise and the resulting solution was stirred for $0.5 \mathrm{~h}$ at $-10^{\circ} \mathrm{C}$ and then for $0.5 \mathrm{~h}$ at $25^{\circ} \mathrm{C}$. The solvents were then removed under vacuum to afford a yellowish solid. Freshly distilled THF was then slowly added under vigorous stirring until the salts were completely dissolved. The freshly prepared $\mathrm{TMPZnCl} \cdot \mathrm{LiCl}$ solution was titrated prior to use at $25^{\circ} \mathrm{C}$ with benzoic acid using 4(phenylazo)diphenylamine1 as indicator. ${ }^{[42]}$

$(\mathrm{TMP})_{2} \mathrm{Zn} \cdot 2 \mathrm{LiCl}$ was prepared as follows in a nearly quantitative yield. A dry and argon flushed $250 \mathrm{~mL}$ Schlenk flask, equipped with a magnetic stirrer and a septum, was charged with 2,2,6,6-tetramethylpiperidine $(10.2 \mathrm{~mL}, 60 \mathrm{mmol})$ and THF $(60 \mathrm{~mL})$. The solution was cooled to $-40^{\circ} \mathrm{C}$ before dropwise addition of BuLi (2.4 M in hexane, $25 \mathrm{~mL}, 60 \mathrm{mmol})$. Then, the reaction mixture was allowed to warm up slowly to $-10^{\circ} \mathrm{C}$ for $1 \mathrm{~h} . \mathrm{ZnCl}_{2}(1.0 \mathrm{M}$ in THF, $30 \mathrm{~mL}, 30 \mathrm{mmol})$ was added dropwise and the resulting solution was stirred for $0.5 \mathrm{~h}$ at $-10^{\circ} \mathrm{C}$ and then for $0.5 \mathrm{~h}$ at $25^{\circ} \mathrm{C}$. The solvents were then removed under vacuum. Freshly distilled THF was then slowly added to the residue under vigorous stirring until the salts were completely dissolved and an orange solution obtained.

TMPMgCl.LiCl was prepared as follows. ${ }^{[26]}$ In a dry and argon flushed Schlenk-flask, 2,2,6,6-tetramethylpiperidine $(14.8 \mathrm{~g}, 105 \mathrm{mmol})$ was added to iPrMgCl. $\mathrm{LiCl}\left(71.4 \mathrm{~mL}, 0.10 \mathrm{~mol}, 1.4 \mathrm{M}\right.$ in THF) at $25^{\circ} \mathrm{C}$ and the mixture was stirred for 3 days at $25^{\circ} \mathrm{C}$. The freshly prepared TMPMgCl. $\mathrm{LiCl}$ was titrated prior to use at $0{ }^{\circ} \mathrm{C}$ with benzoic acid using 4(phenylazo)diphenylamine as indicator. ${ }^{[42]}$

$\mathrm{CoCl}_{2} \cdot 2 \mathrm{LiCl}$ was prepared as follows. ${ }^{[17]} \mathrm{A}$ dry and argon-flushed $250 \mathrm{~mL}$ Schlenk-flask, equipped with a magnetic stirring bar and a rubber septum, was charged with anhydrous $\mathrm{LiCl}(5.9 \mathrm{~g}, 0.14 \mathrm{~mol})$ and heated to $150{ }^{\circ} \mathrm{C}$ under high vacuum for $5 \mathrm{~h}$. After cooling to room temperature under vacuum, anhydrous $\mathrm{CoCl}_{2}(9.1 \mathrm{~g}, 70 \mathrm{mmol})$ was added under argon. The Schlenk-flask was further heated to $130^{\circ} \mathrm{C}$ for $3 \mathrm{~h}$ under high vacuum, cooled to $25^{\circ} \mathrm{C}$ and charged with dry THF $(70 \mathrm{~mL})$. The mixture was vigorously stirred until all solids were dissolved (ca. $8 \mathrm{~h}$ ). The reagent $\mathrm{CoCl}_{2} \cdot 2 \mathrm{LiCl}(1 \mathrm{M}$ in THF) is obtained as a dark blue solution.

1,5-Naphthyridine, ${ }^{[22]}$ activated $\mathrm{Cu},{ }^{[43]}$ methyl (2-aminothiophene-3carboxylate $^{[44]}$ and 2-iodobenzofuran ${ }^{[45]}$ were prepared as reported previously.

All reagents not listed in the publication (main paper or supporting information) were obtained from commercial sources.

\section{Crystallographic data}

CCDC 1896366 (1d), 1896367 (2a') and 1896368 (10ab) contain the crystallographic data for this paper. These data can be obtained free of charge from The Cambridge Crystallographic Data Centre.

The samples were studied with monochromatized Mo-K $\alpha$ radiation $(\lambda=$ $0.71073 \AA$, multilayer monochromator). The $\mathrm{X}$-ray diffraction data of the compounds 1d, 2a' and 10ab were collected at 150(2) K by using a D8 VENTURE Bruker AXS diffractometer equipped with a (CMOS) PHOTON 
100 detector. The structure was solved by dual-space algorithm using the SHELXT program, ${ }^{[46]}$ and then refined with full-matrix least-square methods based on $F^{2}$ (SHELXL-2014). ${ }^{[47]}$ All non-hydrogen atoms were refined with anisotropic atomic displacement parameters. $\mathrm{H}$ atoms were finally included in their calculated positions and treated as riding on their parent atom with constrained thermal parameters. The molecular diagrams were generated by ORTEP-3 (version 2.02). ${ }^{[48]}$

\section{General procedure 1 for the $N$-benzoyloxylation of secondary} amines.

To dibasic potassium phosphate $\left(\mathrm{K}_{2} \mathrm{HPO}_{4} ; 1.3 \mathrm{~g}, 7.5 \mathrm{mmol}\right)$ and freshly dried benzoyl peroxide $(1.2 \mathrm{~g}, 5.0 \mathrm{mmol})$ in DMF $(20 \mathrm{~mL})$ under argon was slowly added the secondary amine $(6.0 \mathrm{mmol})$. The reaction mixture was stirred at room temperature until disappearance of benzoyl peroxide (about $12 \mathrm{~h}$, as shown by TLC monitoring). After addition of saturated aqueous ammonium chloride $(30 \mathrm{~mL})$, extraction was performed using AcOEt $(3 \times 20 \mathrm{~mL})$. The organic phase was washed with saturated aqueous $\mathrm{NaHCO}_{3}(20 \mathrm{~mL})$ and brine $(20 \mathrm{~mL})$, and then dried over $\mathrm{Na}_{2} \mathrm{SO}_{4}$. Removal of the solvent and purification by chromatography on silica gel (the eluent is given in the product description) led to the expected compound.

\section{$N$-(Benzoyloxy)diallylamine.}

The general procedure 1 using diallylamine $(0.74 \mathrm{~mL})$ gave $N$ (benzoyloxy)diallylamine (eluent: hexanes-AcOEt $80: 20 ; R_{f}=0.67$ ) in $76 \%$ yield $(0.83 \mathrm{~g})$ as a yellow oil. ${ }^{1} \mathrm{H} \mathrm{NMR}\left(300 \mathrm{MHz}, \mathrm{CDCl}_{3}\right) \delta 3.39(\mathrm{~d}$, $2 \mathrm{H}, J=6.5 \mathrm{~Hz}$ ), 4.90 (dd, $2 \mathrm{H}, J=10.2$ and $1.6 \mathrm{~Hz}$ ), 5.00 (dd, $2 \mathrm{H}, J=$ 17.2 and $1.6 \mathrm{~Hz}$ ), 5.76 (ddt, $2 \mathrm{H}, J=16.8,10.1$ and $6.5 \mathrm{~Hz}), 7.12(\mathrm{dd}, 2 \mathrm{H}$, $J=8.3$ and $6.9 \mathrm{~Hz}$ ), $7.24(\mathrm{tt}, 1 \mathrm{H}, J=7.4$ and $1.9 \mathrm{~Hz}$ ), $7.72(\mathrm{dd}, 2 \mathrm{H}, J=$ 8.3, $1.4 \mathrm{~Hz})$. The analyses are as described previously. ${ }^{[17]}{ }^{13} \mathrm{C}$ NMR $(75$ $\left.\mathrm{MHz}, \mathrm{CDCl}_{3}\right) \delta 61.7\left(2 \mathrm{CH}_{2}\right), 119.5\left(2 \mathrm{CH}_{2}\right), 128.3(2 \mathrm{CH}), 129.2(\mathrm{C}), 129.4$ $(2 \mathrm{CH}), 132.5(2 \mathrm{CH}), 133.0(\mathrm{CH}), 165.3(\mathrm{C}, \mathrm{C}=\mathrm{O})$.

\section{$N$-(Benzoyloxy)dibutylamine.}

The general procedure 1 using dibutylamine $(1.01 \mathrm{~mL})$ gave $N$ (benzoyloxy)dibutylamine (eluent: hexanes-AcOEt $95: 5 ; R_{f}=0.62$ ) in $59 \%$ yield $(0.74 \mathrm{~g})$ as a colorless oil. ${ }^{1} \mathrm{H} \mathrm{NMR}\left(300 \mathrm{MHz}, \mathrm{CDCl}_{3}\right) \delta 0.86(\mathrm{t}$, $3 \mathrm{H}, J=7.3 \mathrm{~Hz}$ ), 1.34 (sext, $2 \mathrm{H}, J=7.3 \mathrm{~Hz}$ ), 1.54 (ddd, $2 \mathrm{H}, J=15.1,8.7$ and $6.1 \mathrm{~Hz}), 2.92(\mathrm{~d}, 1 \mathrm{H}, J=7.4 \mathrm{~Hz}), 2.94(\mathrm{~d}, 1 \mathrm{H}, J=7.4 \mathrm{~Hz}), 7.39(\mathrm{t}, 2 \mathrm{H}$ $J=7.4 \mathrm{~Hz}), 7.51(\mathrm{tt}, 1 \mathrm{H}, J=7.4$ and $1.9 \mathrm{~Hz}), 7.99(\mathrm{~d}, J=7.1 \mathrm{~Hz}, 1 \mathrm{H})$. The ${ }^{1} \mathrm{H}$ NMR data are as described previously. ${ }^{[49]}{ }^{13} \mathrm{C}$ NMR $(75 \mathrm{MHz}$, $\left.\mathrm{CDCl}_{3}\right) \delta 13.9\left(2 \mathrm{CH}_{3}\right), 20.5\left(2 \mathrm{CH}_{2}\right), 29.0\left(2 \mathrm{CH}_{2}\right), 59.5\left(2 \mathrm{CH}_{2}\right), 128.4$ $(2 \mathrm{CH}), 129.4(\mathrm{C}), 129.5(2 \mathrm{CH}), 132.9(\mathrm{CH}), 165.6(\mathrm{C}, \mathrm{C}=\mathrm{O})$.

\section{$N$-(Benzoyloxy)morpholine.}

The general procedure 1 using morpholine $(0.52 \mathrm{~mL})$ gave $N$ (benzoyloxy)morpholine (eluent: hexanes-AcOEt $50: 50 ; R_{f}=0.62$ ) in $37 \%$ yield $(0.39 \mathrm{~g})$ as a white powder: $\mathrm{mp} 82^{\circ} \mathrm{C}$ (lit. ${ }^{[50]} 81-82{ }^{\circ} \mathrm{C}$ ); IR (ATR): $677,708,793,857,1008,1023,1049,1066,1083,1100,1247$, 1316, 1386, 1453, 1600, 1728, 2849, $2966 \mathrm{~cm}^{-1} ;{ }^{1} \mathrm{H}$ NMR $(300 \mathrm{MHz}$, $\left.\mathrm{CDCl}_{3}\right) \delta$ 2.99-3.01 (m, 2H), 3.39-3.41 (m, 2H), 3.78-3.91 (m, 4H), $7.40(\mathrm{t}$, $2 \mathrm{H}, J=7.6 \mathrm{~Hz}), 7.52(\mathrm{t}, 1 \mathrm{H}, J=7.3 \mathrm{~Hz}), 7.97(\mathrm{~d}, 2 \mathrm{H}, J=7.2 \mathrm{~Hz}) .{ }^{13} \mathrm{C}$ NMR $\left(75 \mathrm{MHz}, \mathrm{CDCl}_{3}\right) \delta 56.9\left(2 \mathrm{CH}_{2}\right), 65.8\left(2 \mathrm{CH}_{2}\right), 128.4(2 \mathrm{CH}), 129.0$ (C), $129.4(2 \mathrm{CH}), 133.2(\mathrm{CH}), 164.5(\mathrm{C}, \mathrm{C}=\mathrm{O})$.

\section{$N$-(Benzoyloxy)piperidine.}

The general procedure 1 using piperidine $(0.59 \mathrm{~mL})$ gave $N$ (benzoyloxy)piperidine (eluent: hexanes-AcOEt 70:30; $R_{f}=0.75$ ) in 64\% yield $(0.66 \mathrm{~g})$ as a beige powder: $\mathrm{mp} 65^{\circ} \mathrm{C}$ (lit. $\left.{ }^{[50]} 62.5-63^{\circ} \mathrm{C}\right)$; IR (ATR): 428, 675, 713, 782, 850, 917, 1019, 1037, 1070, 1092, 1184, 1235, 1249, 1270, 1280, 1319, 1453, 1601, 1728, 2847, $2940 \mathrm{~cm}^{-1} ;{ }^{1} \mathrm{H}$ NMR (300 $\left.\mathrm{MHz} \mathrm{CDCl}_{3}\right) \delta 1.00-1.59(\mathrm{~m}, 6 \mathrm{H}), 2.55(\mathrm{br} \mathrm{s}, 2 \mathrm{H}), 3.28(\mathrm{br} \mathrm{s}, 2 \mathrm{H}), 7.18(\mathrm{t}$, $2 \mathrm{H}, J=7.7 \mathrm{~Hz}), 7.30$ (t, $1 \mathrm{H}, J=7.3 \mathrm{~Hz}), 7.79(\mathrm{~d}, 2 \mathrm{H}, J=7.8 \mathrm{~Hz})$.

\section{N-(Benzoyloxy)diisopropylamine.}

The general procedure 1 using diisopropylamine $(0.84 \mathrm{~mL})$ gave $N$ (benzoyloxy)diisopropylamine (eluent: hexanes-AcOEt 90:10; $R_{f}=0.30$ ) in $45 \%$ yield $(0.50 \mathrm{~g})$ as a yellow oil: IR (ATR): $644,663,708,729,874$, 913, 1025, 1061, 1081, 1177, 1243, 1314, 1383, 1451, 1601, 1740, 2226, 2877, 2938, $2978 \mathrm{~cm}^{-1}$; ${ }^{1} \mathrm{H}$ NMR $\left(300 \mathrm{MHz} \mathrm{CDCl}_{3}\right) \delta 1.03(\mathrm{~d}, 12 \mathrm{H}, J=$ $6.3 \mathrm{~Hz}$ ), 3.28 (hept, $2 \mathrm{H}, J=6.2 \mathrm{~Hz}$ ), 7.31 (t, $2 \mathrm{H}, J=7.3 \mathrm{~Hz}), 7.43(\mathrm{t}, 1 \mathrm{H}, J$ $=7.3 \mathrm{~Hz}), 7.91(\mathrm{~d}, 2 \mathrm{H}, J=7.7 \mathrm{~Hz})$. The NMR data are as described previously. ${ }^{[51]}{ }^{13} \mathrm{C}$ NMR $\left(75 \mathrm{MHz}, \mathrm{CDCl}_{3}\right) \delta 17.2$ (br s, $\left.2 \mathrm{CH}_{3}\right), 20.1$ (br s, $\left.2 \mathrm{CH}_{3}\right), 53.3(2 \mathrm{CH}), 128.3(2 \mathrm{CH}), 129.1(\mathrm{C}), 129.3(2 \mathrm{CH}), 132.8(\mathrm{CH})$, $166.1(\mathrm{C}, \mathrm{C}=\mathrm{O})$.

\section{N-(Benzoyloxy)di(2-methoxyethyl)amine.}

The general procedure 1 using di(2-methoxyethyl)amine $(0.89 \mathrm{~mL})$ gave $\mathrm{N}$-(benzoyloxy)di(2-methoxyethyl)amine (eluent: hexanes-AcOEt 50:50) in $76 \%$ yield $(0.96 \mathrm{~g})$ as a colorless oil: IR (ATR): 1022, 1056, 1116, 1198, 1241, 1450, 1740, $2888 \mathrm{~cm}^{-1} ;{ }^{1} \mathrm{H}$ NMR (400 MHz, $\left.\mathrm{CDCl}_{3}\right) \delta 3.26$ (t, 4H, J $=5.6 \mathrm{~Hz}), 3.29(\mathrm{~s}, 6 \mathrm{H}), 3.61(\mathrm{t}, 4 \mathrm{H}, J=5.8 \mathrm{~Hz}), 7.43-7.45(\mathrm{~m}, 2 \mathrm{H}), 7.57(\mathrm{t}$, $1 \mathrm{H}, J=7.4 \mathrm{~Hz}$ ), $8.02(\mathrm{dd}, 2 \mathrm{H}, J=8.3$ and $1.2 \mathrm{~Hz}) ;{ }^{13} \mathrm{C} \mathrm{NMR}(101 \mathrm{MHz}$, $\left.\mathrm{CDCl}_{3}\right) \delta 59.0\left(2 \mathrm{CH}_{3}\right), 59.4\left(2 \mathrm{CH}_{2}\right), 69.9\left(2 \mathrm{CH}_{2}\right), 128.5(2 \mathrm{CH}), 129.6(\mathrm{C})$, $129.7(2 \mathrm{CH}), 133.2(\mathrm{CH}), 165.6(\mathrm{C}, \mathrm{C}=\mathrm{O})$; MS (El, $70 \mathrm{eV}) \mathrm{m} / \mathrm{z}(\%) 42$ (87), 44 (36), 51 (10), 56 (10), 59 (13), 76 (37), 88 (16), 104 (100), 105 (11), 121 (19), 208 (29); HRMS (EI) $\mathrm{m} / z$ calcd for $\mathrm{C}_{13} \mathrm{H}_{19} \mathrm{NO}_{4}$ : 253.1314; found: 253.1307.

\section{N-(Benzoyloxy)-1,4-dioxa-8-azaspiro[4.5]decane.}

The general procedure 1 using 1,4-dioxa-8-azaspiro[4.5]decane (0.77 mL) gave $\mathrm{N}$-(benzoyloxy)-1,4-dioxa-8-azaspiro[4.5]decane (eluent: hexanes-AcOEt $50: 50)$ in $71 \%$ yield $(0.93 \mathrm{~g})$ as a white solid: IR (ATR): 1060, 1081, 1122, 1245, 1447, 1733, $2959 \mathrm{~cm}^{-1} ;{ }^{1} \mathrm{H}$ NMR $(400 \mathrm{MHz}$, $\left.\mathrm{CDCl}_{3}\right) \delta 1.97(\mathrm{t}, 4 \mathrm{H}, \mathrm{J}=5.7 \mathrm{~Hz}$ ), 3.27 (br s, 2H), 3.45 (br s, 2H), 4.00 (s, $\left.4 \mathrm{H}, \mathrm{OCH}_{2} \mathrm{CH}_{2} \mathrm{O}\right), 7.44(\mathrm{t}, 2 \mathrm{H}, J=7.5 \mathrm{~Hz}), 7.57(\mathrm{t}, 1 \mathrm{H}, J=7.5 \mathrm{~Hz}) ;{ }^{13} \mathrm{C}$ NMR $\left(101 \mathrm{MHz}, \mathrm{CDCl}_{3}\right) \delta 32.8\left(2 \mathrm{CH}_{2}\right), 54.1\left(2 \mathrm{CH}_{2}\right), 64.6\left(2 \mathrm{CH}_{2}\right), 106.3$ (C), $128.5(2 \mathrm{CH}), 129.6(2 \mathrm{CH}), 129.6(\mathrm{C}), 133.1(\mathrm{CH}), 164.9(\mathrm{C}, \mathrm{C}=\mathrm{O})$ The NMR data are as described previously. ${ }^{[52]} \mathrm{MS}(\mathrm{El}, 70 \mathrm{eV}) \mathrm{m} / \mathrm{z}(\%) 42$ (11), 43 (100), 45 (14), 50 (14), 61 (12), 77 (49), 88 (11), 87 (13), 122 (55); HRMS (El) $m / z$ calcd for $\mathrm{C}_{14} \mathrm{H}_{17} \mathrm{NO}_{4}$ : 263.1158; found: 263.1156.

\section{$N$-(Benzoyloxy)azepane.}

The general procedure 1 using azepane $(0.68 \mathrm{~mL})$ gave $N$ (benzoyloxy)azepane as reported previously. ${ }^{[53]}{ }^{1} \mathrm{H}$ NMR $(400 \mathrm{MHz}$, $\left.\mathrm{CDCl}_{3}\right) \delta 1.68(\mathrm{dt}, 4 \mathrm{H}, J=6.1$ and $3.0 \mathrm{~Hz}$ ), 1.82 (ddd, $4 \mathrm{H}, J=6.8,4.4$ and $1.8 \mathrm{~Hz}), 3.33(\mathrm{t}, 4 \mathrm{H}, J=5.5 \mathrm{~Hz}), 7.43(\mathrm{t}, 2 \mathrm{H}, J=7.7 \mathrm{~Hz}), 7.55(\mathrm{t}, 1 \mathrm{H}, J=$ $7.4 \mathrm{~Hz}), 8.00(\mathrm{dd}, 2 \mathrm{H}, J=8.3$ and $1.4 \mathrm{~Hz}) ;{ }^{13} \mathrm{C} \mathrm{NMR}\left(101 \mathrm{MHz}, \mathrm{CDCl}_{3}\right) \delta$ $24.2\left(2 \mathrm{CH}_{2}\right), 26.5\left(2 \mathrm{CH}_{2}\right), 59.6\left(2 \mathrm{CH}_{2}\right), 128.5(2 \mathrm{CH}), 129.5(2 \mathrm{CH}), 129.8$ $(\mathrm{C}), 133.0(\mathrm{CH}), 164.9(\mathrm{C}, \mathrm{C}=\mathrm{O})$.

\section{N-(Benzoyloxy)-1,2,3,4-tetrahydroisoquinoline.}

The general procedure 1 using 1,2,3,4-tetrahydroisoquinoline $(0.76 \mathrm{~mL})$ gave $N$-(benzoyloxy)-1,2,3,4-tetrahydroisoquinoline as reported previously. ${ }^{[18]}{ }^{1} \mathrm{H}$ NMR $\left(400 \mathrm{MHz} \mathrm{CDCl}_{3}\right) \delta 3.12(\mathrm{t}, 2 \mathrm{H}, J=6.0 \mathrm{~Hz}), 3.55$ (br s, 2H), 4.43 (br s, 2H, H1'), $7.06(\mathrm{~d}, 1 \mathrm{H}, J=6.4 \mathrm{~Hz}), 7.12-7.23(\mathrm{~m}, 3 \mathrm{H})$, 
$7.42(\mathrm{t}, 2 \mathrm{H}, J=7.7 \mathrm{~Hz}), 7.55$ (t, $1 \mathrm{H}, J=7.4 \mathrm{~Hz}), 8.00(\mathrm{~d}, 2 \mathrm{H}, J=7.1 \mathrm{~Hz})$ ${ }^{13} \mathrm{C}$ NMR $\left(101 \mathrm{MHz}, \mathrm{CDCl}_{3}\right) \delta 26.7\left(\mathrm{CH}_{2}\right), 53.4\left(\mathrm{CH}_{2}\right), 58.1\left(\mathrm{CH}_{2}\right), 126.3$ $(\mathrm{CH}), 126.8(\mathrm{CH}), 126.9(\mathrm{CH}), 128.4(\mathrm{CH}), 128.4(2 \mathrm{CH}), 129.3(\mathrm{C}), 129.5$ (2CH), $132.3(\mathrm{C}), 132.9(\mathrm{C}), 133.1(\mathrm{CH}), 164.9(\mathrm{C}, \mathrm{C}=\mathrm{O})$.

\section{General procedure 2 for the deprotolithiation-zincation-amination of benzothiophene.}

To a stirred mixture of benzothiophene $(0.20 \mathrm{~g}, 1.5 \mathrm{mmol})$ and $\mathrm{ZnCl}_{2} \cdot \operatorname{TMEDA}(0.39 \mathrm{~g}, 1.5 \mathrm{mmol})$ in THF $(3 \mathrm{~mL})$ at $-20^{\circ} \mathrm{C}$ was added dropwise a THF-hexane solution of LiTMP $(1.5 \mathrm{mmol})$ to a stirred, cooled $\left(0^{\circ} \mathrm{C}\right)$ solution of 2,2,6,6-tetramethylpiperidine $(0.28 \mathrm{~mL}, 1.7 \mathrm{mmol})$ in THF ( $3 \mathrm{~mL}$ ) and stirring for $5 \mathrm{~min}$ ) cooled at $-20^{\circ} \mathrm{C}$. After $0.5 \mathrm{~h}$ at $-20^{\circ} \mathrm{C}$, the solution was added dropwise to the $\mathrm{N}$-benzoyloxy secondary amine $(1.2 \mathrm{mmol})$ and anhydrous copper(II) acetate $(14 \mathrm{mg}, 75 \mu \mathrm{mol})$ in THF $(3 \mathrm{~mL})$ at $-20^{\circ} \mathrm{C}$. The mixture was stirred overnight before addition of an aqueous saturated solution of $\mathrm{Na}_{2} \mathrm{CO}_{3}(5 \mathrm{~mL})$ and extraction with $\mathrm{Et}_{2} \mathrm{O}(3$ $x 20 \mathrm{~mL}$ ). The combined organic layers were dried over $\mathrm{MgSO}_{4}$, filtered and concentrated under reduced pressure. The crude product was purified by chromatography over silica gel (the eluent is given in the product description).

\section{2-(Diallylamino)benzothiophene (1a).}

The general procedure 2 using $N$-(benzoyloxy)diallylamine $(0.26 \mathrm{~g})$ gave 1a (eluent: hexanes- $\mathrm{CH}_{2} \mathrm{Cl}_{2}$ 95:5; $\mathrm{R}_{\mathrm{f}}=0.42$ ) in $52 \%$ yield $(0.14 \mathrm{~g}$ ) as a yellow oil: IR (ATR): 566, 704, 736, 794, 863, 923, 1016, 1065, 1184, 1258, 1355, 1416, 1439, 1540, 1563, 1640, $2962 \mathrm{~cm}^{-1}$; ${ }^{1} \mathrm{H}$ NMR (300 $\left.\mathrm{MHz}, \mathrm{CDCl}_{3}\right) \delta 3.97(\mathrm{dt}, 4 \mathrm{H}, J=5.6$ and $1.5 \mathrm{~Hz}$ ), $5.26(\mathrm{dd}, 2 \mathrm{H}, J=8.9$ and $1.5 \mathrm{~Hz}$ ), $5.33(\mathrm{dd}, 2 \mathrm{H}, J=17.3$ and $1.6 \mathrm{~Hz}$ ), 5.92 (ddt, $2 \mathrm{H}, J=17.0$, 10.1 and $5.6 \mathrm{~Hz}), 6.06(\mathrm{~s}, 1 \mathrm{H}, \mathrm{H} 3), 7.06(\mathrm{t}, 1 \mathrm{H}, J=7.6 \mathrm{~Hz}), 7.24(\mathrm{t}, 1 \mathrm{H}, J$ $=7.6 \mathrm{~Hz}), 7.43(\mathrm{dd}, 1 \mathrm{H}, J=8.0$ and $1.1 \mathrm{~Hz}), 7.59(\mathrm{~d}, 1 \mathrm{H}, J=7.9 \mathrm{~Hz}) ;{ }^{13} \mathrm{C}$ NMR $\left(75 \mathrm{MHz}, \mathrm{CDCl}_{3}\right) \delta 54.9\left(2 \mathrm{CH}_{2}\right), 96.5(\mathrm{CH}), 117.4\left(2 \mathrm{CH}_{2}\right), 120.0$ $(\mathrm{CH}), 120.3(\mathrm{CH}), 121.2(\mathrm{CH}), 124.4(\mathrm{CH}), 131.8(\mathrm{C}), 133.0(2 \mathrm{CH}), 141.4$ (C), 155.7 (C). HRMS (EI) $\mathrm{m} / \mathrm{z}$ calcd for $\mathrm{C}_{14} \mathrm{H}_{15} \mathrm{NS}$ : 229.0925; found: 229.0921. 2,2'-Bi(benzothiophene) (1') was also isolated. This compound could be obtained in $25 \%$ yield by performing the same procedure without O-benzoyl hydroxylamine: white powder; $\mathrm{mp} 262{ }^{\circ} \mathrm{C}$ (lit. ${ }^{[54]} 260$ $261^{\circ} \mathrm{C}$ ); IR (ATR): 476, 557, 722, 736, 814, 937, 1013, 1067, 1177, 1250 $1420,1451,1711,1944,2851,2923,3053 \mathrm{~cm}^{-1} ;{ }^{1} \mathrm{H}$ NMR $(300 \mathrm{MHz}$, $\left.\mathrm{CDCl}_{3}\right) \delta$ 7.30-7.39 (m, 4H), 7.52 (s, 2H), 7.74-7.84 (m, 4H); ${ }^{13} \mathrm{C}$ NMR $(75$ $\left.\mathrm{MHz}, \mathrm{CDCl}_{3}\right) \delta 121.6(\mathrm{CH}), 122.3(\mathrm{CH}), 123.9(\mathrm{CH}), 124.9(\mathrm{CH}), 125.1$ $(\mathrm{CH}), 137.4(\mathrm{C}), 139.6(\mathrm{C}), 140.3(\mathrm{C})$.

2-(Dibutylamino)benzothiophene (1b).

The general procedure 2 using $\mathrm{N}$-(benzoyloxy)dibutylamine $(0.30 \mathrm{~g})$ gave 1b (eluent: hexanes- $\mathrm{Et}_{3} \mathrm{~N}$ 95:5; $\mathrm{R}_{\mathrm{f}}=0.67$ ) in $63 \%$ yield $(0.25 \mathrm{~g})$ as a yellow oil: IR (ATR): 726, 756, 919, 1018, 1065, 1111, 1133, 1194, 1243, 1279, 1311, 1369, 1440, 1539, 1562, 2861, 2928, $2955 \mathrm{~cm}^{-1}$; ${ }^{1} \mathrm{H}$ NMR $\left(300 \mathrm{MHz}, \mathrm{CDCl}_{3}\right) \delta 0.96\left(\mathrm{t}, 6 \mathrm{H}, J=7.3 \mathrm{~Hz}, \mathrm{CH}_{3}\right), 1.38$ (dq, $4 \mathrm{H}, J=14.6$ and $7.0 \mathrm{~Hz}, \mathrm{CH}_{2}$ ), 1.65 (quintuplet, $4 \mathrm{H}, J=7.5 \mathrm{~Hz}, \mathrm{CH}_{2}$ ), 3.28 (t, $4 \mathrm{H}, J=$ $\left.7.5 \mathrm{~Hz}, \mathrm{CH}_{2}\right), 5.88(\mathrm{~s}, 1 \mathrm{H}, \mathrm{H} 3), 6.97(\mathrm{t}, 1 \mathrm{H}, J=7.5 \mathrm{~Hz}), 7.18(\mathrm{t}, 1 \mathrm{H}, J=$ $7.5 \mathrm{~Hz}), 7.36(\mathrm{~d}, 1 \mathrm{H}, J=7.9 \mathrm{~Hz}), 7.53(\mathrm{~d}, 1 \mathrm{H}, J=7.9 \mathrm{~Hz}) ;{ }^{13} \mathrm{C}$ NMR $(75$ $\left.\mathrm{MHz}, \mathrm{CDCl}_{3}\right) \delta 14.0\left(2 \mathrm{CH}_{3}\right), 20.4\left(2 \mathrm{CH}_{2}\right), 29.5\left(2 \mathrm{CH}_{2}\right), 53.2\left(2 \mathrm{CH}_{2}\right), 94.9$ $(\mathrm{CH}), 119.6(\mathrm{CH}), 119.8(\mathrm{CH}), 121.3(\mathrm{CH}), 124.5(\mathrm{CH}), 131.6(\mathrm{C}), 141.9$ (C), 156.3 (C). HRMS (EI) $\mathrm{m} / z$ calcd for $\mathrm{C}_{16} \mathrm{H}_{23} \mathrm{NS}$ : 261.1551; found: 261.1549 .

\section{2-(Morpholino)benzothiophene (1c).}

The general procedure 2 using $N$-(benzoyloxy)morpholine $(0.25 \mathrm{~g})$ gave 1c (eluent: hexanes- $\left.\mathrm{Et}_{3} \mathrm{~N} 98: 2 ; \mathrm{R}_{\mathrm{f}}=0.30\right)$ in $29 \%$ yield $(76 \mathrm{mg}$ ) as a beige powder: $\mathrm{mp} 180^{\circ} \mathrm{C}\left(\right.$ lit. $\left.{ }^{[10 a]} 180-181.5^{\circ} \mathrm{C}\right)$; IR (ATR): $569,592,653$, $723,744,780,868,901,1013,1065,1116,1186,1212,1250,1264$, 1303, 1375, 1437, 1455, 1527, 1557, 2850, $2958 \mathrm{~cm}^{-1} ;{ }^{1} \mathrm{H}$ NMR (300 $\left.\mathrm{MHz}, \mathrm{CDCl}_{3}\right) \delta 3.24$ (t, $4 \mathrm{H}, J=4.9 \mathrm{~Hz}$ ), $3.86(\mathrm{t}, 4 \mathrm{H}, J=4.8 \mathrm{~Hz}), 6.23(\mathrm{~s}$ $1 \mathrm{H}, \mathrm{H} 3), 7.10(\mathrm{t}, 1 \mathrm{H}, J=7.6 \mathrm{~Hz}), 7.25(\mathrm{t}, 1 \mathrm{H}, J=7.4 \mathrm{~Hz}), 7.48(\mathrm{~d}, 1 \mathrm{H}, J=$ $7.9 \mathrm{~Hz}), 7.61(\mathrm{~d}, 1 \mathrm{H}, J=7.9 \mathrm{~Hz}) ;{ }^{13} \mathrm{C}$ NMR $\left(75 \mathrm{MHz}, \mathrm{CDCl}_{3}\right) \delta 51.1$ $\left(2 \mathrm{CH}_{2}\right), 66.4\left(2 \mathrm{CH}_{2}\right), 99.6(\mathrm{CH}), 121.2(\mathrm{CH}), 121.7(\mathrm{CH}), 121.8(\mathrm{CH})$, $124.7(\mathrm{CH}), 132.8(\mathrm{C}), 140.4(\mathrm{C}), 157.9(\mathrm{C})$. These data are as reported previously. ${ }^{[10 a]}$

\section{2-(Piperidino)benzothiophene (1d)}

The general procedure 2 using $N$-(benzoyloxy)piperidine $(0.25 \mathrm{~g})$ gave 1d (eluent: hexanes- $\left.E t_{3} \mathrm{~N} 98: 2 ; R_{f}=0.40\right)$ in $68 \%$ yield $(0.18 \mathrm{~g})$ as a white powder: $\mathrm{mp} 102{ }^{\circ} \mathrm{C}$ (lit. ${ }^{[55]} 99-100{ }^{\circ} \mathrm{C}$ ); IR (ATR): $479,558,581,641,721$ $734,772,812,855,1009,1069,1117,1203,1236,1255,1316,1384$, 1439, 1532, 1559, 1717, 2935, $3055 \mathrm{~cm}^{-1} ;{ }^{1} \mathrm{H}$ NMR $\left(300 \mathrm{MHz}, \mathrm{CDCl}_{3}\right) \delta$ $1.61-1.67(\mathrm{~m}, 2 \mathrm{H}), 1.73-1.80(\mathrm{~m}, 4 \mathrm{H}), 3.28(\mathrm{t}, 4 \mathrm{H}, J=5.5 \mathrm{~Hz}), 6.18(\mathrm{~s}, 1 \mathrm{H}$, H3), $7.08(\mathrm{t}, 1 \mathrm{H}, J=7.5 \mathrm{~Hz}), 7.24(\mathrm{t}, 1 \mathrm{H}, J=7.5 \mathrm{~Hz}), 7.46$ (d, $1 \mathrm{H}, J=7.6$ $\mathrm{Hz}), 7.61(\mathrm{~d}, J=7.9 \mathrm{~Hz}, 1 \mathrm{H}) ;{ }^{13} \mathrm{C}$ NMR $\left(75 \mathrm{MHz}, \mathrm{CDCl}_{3}\right) \delta 24.0\left(\mathrm{CH}_{2}\right)$, $25.3\left(2 \mathrm{CH}_{2}\right), 52.1\left(2 \mathrm{CH}_{2}\right), 98.5(\mathrm{CH}), 120.7(\mathrm{CH}), 121.0(\mathrm{CH}), 121.6(\mathrm{CH})$, $124.5(\mathrm{CH}), 132.7(\mathrm{C}), 141.0(\mathrm{C}), 158.6(\mathrm{C})$. Crystal data for 1d. $\mathrm{C}_{13} \mathrm{H}_{15} \mathrm{NS}, \mathrm{M}=217.32$, triclinic, $P-1, a=6.0747(7), b=12.9937(15), c=$ 14.7777(18) $\AA, \alpha=74.742(4), \beta=82.826(4), \gamma=86.354(4) \circ, V=$ 1115.9(2) $\AA^{3}, Z=4, d=1.293 \mathrm{~g} \mathrm{~cm}^{-3}, \mu=0.255 \mathrm{~mm}^{-1}$. A final refinement on $F^{2}$ with 5071 unique intensities and 297 parameters converged at $\omega R\left(F^{2}\right)=0.0949(R(F)=0.0411)$ for 4457 observed reflections with $I>$ $2 \sigma($ ). CCDC 1896366.

\section{2-Chloro-5-(diallylamino)thiophene (2a).}

The general procedure 2, from 2-chlorothiophene $(0.18 \mathrm{~g})$ instead of thiophene, using $N$-(benzoyloxy)diallylamine $(0.26 \mathrm{~g})$ gave 2a (eluent: hexanes- $\mathrm{Et}_{3} \mathrm{~N}$ 95:5; $\left.\mathrm{R}_{\mathrm{f}}=0.50\right)$ in $49 \%$ yield $(0.13 \mathrm{~g})$ as a colorless oil of low stability (e.g. a solution in $\mathrm{CDCl}_{3}$ becomes dark after a few minutes): ${ }^{1} \mathrm{H}$ NMR $\left(300 \mathrm{MHz}, \mathrm{CDCl}_{3}\right) \delta 3.77(\mathrm{~d}, 1 \mathrm{H}, J=5.8 \mathrm{~Hz}), 5.20(\mathrm{dd}, 2 \mathrm{H}, J=$ 10.0 and $1.3 \mathrm{~Hz}$ ), 5.22 (dd, $2 \mathrm{H}, J=17.2$ and $1.5 \mathrm{~Hz}), 5.71(\mathrm{~d}, 1 \mathrm{H}, J=4.0$ $\mathrm{Hz}$ ), 5.83 (ddt, $2 \mathrm{H}, J=16.0,10.4$ and $5.8 \mathrm{~Hz}), 6.53(\mathrm{~d}, 1 \mathrm{H}, J=4.0 \mathrm{~Hz}$ ). Before the IR spectra and ${ }^{13} \mathrm{C}$ NMR could be recorded, degradation to give allylated products was noticed and formation of 5,5'-dichloro-2,2'bithiophene (2a') was identified by NMR: ${ }^{1} \mathrm{H}$ NMR $\left(300 \mathrm{MHz}, \mathrm{CDCl}_{3}\right) \delta$ $6.82(\mathrm{~d}, 2 \mathrm{H}, J=3.9 \mathrm{~Hz}), 6.85(\mathrm{~d}, 2 \mathrm{H}, J=3.9 \mathrm{~Hz}) ;{ }^{13} \mathrm{C} \mathrm{NMR}(75 \mathrm{MHz}$, $\left.\mathrm{CDCl}_{3}\right) \delta 123.2(2 \mathrm{CH}), 127.0(2 \mathrm{CH}), 129.3(2 \mathrm{C}), 135.2(2 \mathrm{C})$. These NMR data are similar to those reported previously. ${ }^{[56]}$ Crystal data for $2 a^{\prime}$. $\mathrm{C}_{8} \mathrm{H}_{4} \mathrm{Cl}_{2} \mathrm{~S}_{2}, \mathrm{M}=235.13$, orthorhombic, $P c c n, a=7.3894(12), b=$ 21.610(3), $c=5.7035(9) \AA, V=910.8(2) \AA^{3}, Z=4, d=1.715 \mathrm{~g} \mathrm{~cm}^{-3}, \mu=$ $1.104 \mathrm{~mm}^{-1}$. A final refinement on $F^{2}$ with 1041 unique intensities and 55 parameters converged at $\omega R\left(F^{2}\right)=0.1336(R(F)=0.0682)$ for 904 observed reflections with $I>2 \sigma(I)$. CCDC 1896367

\section{4-(Diallylamino)-1,5-naphthyridine (3a) by deprotometalation-} amination of 1,5-naphthyridine.

To a stirred mixture of 1,5 -naphthyridine $(0.13 \mathrm{~g}, 1.0 \mathrm{mmol})$ in THF $(3 \mathrm{~mL})$ at room temperature was added dropwise a solution of TMPZnCl.LiCl (1.04 M THF solution; $1.1 \mathrm{mmol})$. After $2 \mathrm{~h}$ at this temperature, the mixture was treated dropwise by a solution of $N$ (benzoyloxy)diallylamine $(0.18 \mathrm{~g}, 0.8 \mathrm{mmol})$ and anhydrous copper(II) acetate $(9 \mathrm{mg}, 50 \mu \mathrm{mol})$ in THF $(3 \mathrm{~mL})$. It was stirred overnight before addition of an aqueous saturated solution of $\mathrm{NH}_{4} \mathrm{Cl}(4 \mathrm{~mL})$ and extraction with AcOEt $(3 \times 10 \mathrm{~mL})$. The combined organic layers were washed by aqueous saturated solutions of $\mathrm{NaHCO}_{3}(4 \mathrm{~mL})$ and brine $(4 \mathrm{~mL})$, dried over $\mathrm{MgSO}_{4}$, filtered and concentrated under reduced pressure to afford, 
after chromatography over silica gel (eluent: $\left.A c O E t ; R_{f}=0.50\right)$, 3a in $64 \%$ yield $(0.11 \mathrm{~g})$ as a yellow oil: IR (ATR): $555,610,657,712,790,823$ 919, 993, 1111, 1171, 1232, 1249, 1288, 1347, 1411, 1480, 1504, 1563, 1639, 2910, 2980, $3075 \mathrm{~cm}^{-1}$; ${ }^{1} \mathrm{H}$ NMR $\left(300 \mathrm{MHz}, \mathrm{CDCl}_{3}\right) \delta 4.42$ (dd, $4 \mathrm{H}$, $J=4.3$ and $\left.2.8 \mathrm{~Hz}, \mathrm{H} 1^{\prime}\right), 5.19-5.25\left(\mathrm{~m}, 4 \mathrm{H}, \mathrm{H} 3^{\prime}\right), 6.04$ (ddt, $2 \mathrm{H}, J=17.8$, 9.9 and $\left.5.5 \mathrm{~Hz}, \mathrm{H} 2^{\prime}\right), 6.69(\mathrm{~d}, 1 \mathrm{H}, J=5.5 \mathrm{~Hz}, \mathrm{H} 3$ ), 7.50 (dd, $1 \mathrm{H}, J=8.5$ and $4.0 \mathrm{~Hz}, \mathrm{H} 7), 8.23(\mathrm{dd}, 1 \mathrm{H}, J=8.5$ and $1.8 \mathrm{~Hz}, \mathrm{H} 8), 8.50(\mathrm{~d}, 1 \mathrm{H}, J=$ $5.5 \mathrm{~Hz}, \mathrm{H} 2), 8.73(\mathrm{dd}, 1 \mathrm{H}, J=4.1$ and $1.7 \mathrm{~Hz}, \mathrm{H6}) ;{ }^{13} \mathrm{C} \mathrm{NMR}(75 \mathrm{MHz}$, $\left.\mathrm{CDCl}_{3}\right) \delta 54.7\left(2 \mathrm{CH}_{2}\right), 107.1(\mathrm{CH}), 117.4\left(2 \mathrm{CH}_{2}\right), 123.8(\mathrm{CH}), 134.0$ $(2 \mathrm{CH}), 137.4(\mathrm{CH}), 137.9(\mathrm{C}), 145.3(\mathrm{C}), 146.0(\mathrm{CH}), 150.8(\mathrm{CH}), 152.5$ (C). HRMS (EI) $\mathrm{m} / z$ calcd for $\mathrm{C}_{14} \mathrm{H}_{15} \mathrm{~N}_{3}$ : 225.1266; found: 225.1268 .

\section{4-(Diisopropylamino)-1,5-naphthyridine (3b) by deprotometalation- amination of 1,5-naphthyridine.}

To a stirred mixture of 1,5 -naphthyridine $(0.13 \mathrm{~g}, 1.0 \mathrm{mmol})$ in THF $(3 \mathrm{~mL})$ at $-40^{\circ} \mathrm{C}$ was added dropwise a solution of TMPMgCl. LiCl (1.07 M THF solution; $1.1 \mathrm{mmol})$. After $1 \mathrm{~h}$ at this temperature, the mixture was treated dropwise by a solution of $\mathrm{N}$ (benzoyloxy)diisopropylamine $(0.18 \mathrm{~g}, \quad 0.8 \mathrm{mmol})$ and anhydrous copper(II) acetate $(9 \mathrm{mg}, 50 \mu \mathrm{mol})$ in THF $(3 \mathrm{~mL})$. It was slowly warmed to room temperature and stirred overnight before addition of an aqueous saturated solution of $\mathrm{NH}_{4} \mathrm{Cl}(4 \mathrm{~mL})$ and extraction with AcOEt $(3 \times 10 \mathrm{~mL})$. The combined organic layers were washed by aqueous saturated solutions of $\mathrm{NaHCO}_{3}(4 \mathrm{~mL})$ and brine $(4 \mathrm{~mL})$, dried over $\mathrm{MgSO}_{4}$, filtered and concentrated under reduced pressure to afford, after chromatography over silica gel (eluent: AcOEt; $R_{f}=0.27$ ), $3 \mathbf{b}$ in $52 \%$ yield $(95 \mathrm{mg})$ as a yellow oil: ${ }^{1} \mathrm{H} \mathrm{NMR}\left(400 \mathrm{MHz}, \mathrm{CDCl}_{3}\right) \delta 1.42(\mathrm{~d}, 12 \mathrm{H}, J$ $\left.=6.8 \mathrm{~Hz}, \mathrm{CH}_{3}\right), 4.57$ (septuplet, $\left.2 \mathrm{H}, J=6.8 \mathrm{~Hz}\right), 6.95(\mathrm{~d}, 1 \mathrm{H}, J=5.6 \mathrm{~Hz}$, $\mathrm{H} 3$ ), 7.46 (dd, $1 \mathrm{H}, J=8.5$ and $4.0 \mathrm{~Hz}, \mathrm{H} 7$ ), 8.20 (dd, $1 \mathrm{H}, J=8.5$ and 1.7 $\mathrm{Hz}, \mathrm{H} 8), 8.46(\mathrm{~d}, 1 \mathrm{H}, J=5.6 \mathrm{~Hz}, \mathrm{H} 2), 8.71(\mathrm{dd}, 1 \mathrm{H}, J=4.0$ and $1.7 \mathrm{~Hz}$, $\mathrm{H} 6) ;{ }^{13} \mathrm{C}$ NMR $\left(101 \mathrm{MHz}, \mathrm{CDCl}_{3}\right) \delta 21.9\left(4 \mathrm{CH}_{3}\right), 50.5(2 \mathrm{CH}), 110.2(\mathrm{CH})$ $123.6(\mathrm{CH}), 137.2(\mathrm{CH}), 139.7(\mathrm{C}), 145.6(\mathrm{CH}), 145.8(\mathrm{C}), 149.8(\mathrm{CH})$, 152.9 (C). Note that $\mathbf{3 b}$ is contaminated with impurities.

\section{4-Iodo-1,5-naphthyridine (3-I).}

To a stirred mixture of 1,5 -naphthyridine $(0.26 \mathrm{~g}, 2.0 \mathrm{mmol})$ in THF $(4 \mathrm{~mL})$ at room temperature was added dropwise a solution of TMPZnCl.LiCl[22] $(1.04 \mathrm{M}$ THF solution; $2.4 \mathrm{mmol})$. After $2 \mathrm{~h}$ at room temperature, a solution of iodine $(0.76 \mathrm{~g}, 3.0 \mathrm{mmol})$ in THF $(3 \mathrm{~mL})$ was added. The resulting mixture was stirred overnight before addition of an aqueous saturated solution of $\mathrm{Na}_{2} \mathrm{~S}_{2} \mathrm{O}_{3}(5 \mathrm{~mL})$ and extraction with AcOEt $(3 \times 10 \mathrm{~mL})$. The combined organic layers were washed with brine $(5 \mathrm{~mL})$, dried over $\mathrm{Na}_{2} \mathrm{SO}_{4}$, filtered and concentrated under reduced pressure. The crude product was purified by chromatography over silica ge (eluent: hexanes-AcOEt $60: 40)$ to afford $3-1$ in $72 \%$ yield $(0.37 \mathrm{~g})$ as a whitish powder: $\mathrm{mp} 91-93{ }^{\circ} \mathrm{C} ;{ }^{1} \mathrm{H} \mathrm{NMR}\left(300 \mathrm{MHz}, \mathrm{CDCl}_{3}\right) \delta 7.70(\mathrm{dd}, 1 \mathrm{H}$, $J=8.5$ and $4.2 \mathrm{~Hz}, \mathrm{H} 7), 8.28(\mathrm{~d}, 1 \mathrm{H}, J=4.5 \mathrm{~Hz}$ ), 8.37 (dd, $1 \mathrm{H}, J=8.4$ and $1.6 \mathrm{~Hz}, \mathrm{H} 8), 8.53(\mathrm{~d}, 1 \mathrm{H}, J=4.5 \mathrm{~Hz}), 9.06(\mathrm{dd}, 1 \mathrm{H}, J=4.2$ and 1.6 $\mathrm{Hz}, \mathrm{H6}$ ); ${ }^{13} \mathrm{C}$ NMR $\left(75 \mathrm{MHz}, \mathrm{CDCl}_{3}\right) \delta 116.4$ (C, C-I), $125.5(\mathrm{CH}), 135.4$ $(\mathrm{CH}), 138.4(\mathrm{CH}), 143.9(\mathrm{C}), 144.0(\mathrm{C}), 150.8(\mathrm{CH}), 152.2(\mathrm{CH})$. These data are similar to those described previously. ${ }^{[22]}$ Using procedure 3 led to both 3-I (34\% yield) and 4,8-diiodo-1,5-naphthyridine 3'-I. The latter was isolated in $6 \%$ yield as a white powder: $\mathrm{mp}>260^{\circ} \mathrm{C}$; IR (ATR): 491 , $590,630,683,851,1031,1079,1167,1210,1257,1325,1358,1376$, 1398, 1459, 1578, 1695, 1957, 2851, $2921 \mathrm{~cm}^{-1} ;{ }^{1} \mathrm{H}$ NMR $(300 \mathrm{MHz}$, $\left.\mathrm{CDCl}_{3}\right) \delta 8.32(\mathrm{~d}, 2 \mathrm{H}, J=4.5 \mathrm{~Hz}), 8.58(\mathrm{~d}, 2 \mathrm{H}, J=4.5 \mathrm{~Hz}) ;{ }^{13} \mathrm{C} \mathrm{NMR}(75$ $\left.\mathrm{MHz}, \mathrm{CDCl}_{3}\right) \delta 116.6(2 \mathrm{C}, \mathrm{C} 4$ and $\mathrm{C} 8), 136.4(2 \mathrm{CH}, \mathrm{C} 3$ and $\mathrm{C} 7), 143.6$ (2C), 151.5 (2CH, C2 and $\mathrm{C} 6)$.

\section{4-Morpholino-1,5-naphthyridine (3c).}

A degassed sealed tube containing 4-iodo-1,5-naphthyridine (3-I; $96 \mathrm{mg}$, $0.375 \mathrm{mmol})$ and morpholine $(0.195 \mathrm{~mL}, 2.25 \mathrm{mmol})$ in isopropanol $(1 \mathrm{~mL})$ was heated at $110^{\circ} \mathrm{C}$ for $72 \mathrm{~h}$. The cooled residue was poured onto brine and extracted with AcOEt $(3 \times 10 \mathrm{~mL})$. The combined organic layers were dried over $\mathrm{Na}_{2} \mathrm{SO}_{4}$, filtered and concentrated under reduced pressure. The crude product was purified by chromatography over silica gel (eluent: AcOEt; $R_{f}=0.30$ ) to afford $3 c$ in $70 \%$ yield $(57 \mathrm{mg}$ ) as a white powder: $\mathrm{mp} 116-118{ }^{\circ} \mathrm{C}$; IR (ATR): 1026, 1111, 1249, 1265, 1305 , $1372,1414,1477,1503,1566,1604,2864,2966,3205,3350 \mathrm{~cm}^{-1} ;{ }^{1} \mathrm{H}$ NMR $\left(300 \mathrm{MHz}, \mathrm{CDCl}_{3}\right) \delta 3.68-3.71\left(\mathrm{~m}, 4 \mathrm{H}, 2 \mathrm{CH}_{2}\right), 3.97-4.01(\mathrm{~m}, 4 \mathrm{H}$ $\left.2 \mathrm{CH}_{2}\right), 6.84(\mathrm{~d}, 1 \mathrm{H}, J=5.1 \mathrm{~Hz}, \mathrm{H} 3), 7.55(\mathrm{dd}, 1 \mathrm{H}, J=8.5$ and $4.1 \mathrm{~Hz}, \mathrm{H} 7)$, $8.30(\mathrm{dd}, 1 \mathrm{H}, J=8.5$ and $1.8 \mathrm{~Hz}, \mathrm{H} 8), 8.68(\mathrm{~d}, 1 \mathrm{H}, J=5.1 \mathrm{~Hz}, \mathrm{H} 2), 8.81$ (dd, $1 \mathrm{H}, J=4.1$ and $1.7 \mathrm{~Hz}, \mathrm{H} 6) ;{ }^{13} \mathrm{C}$ NMR $\left(75 \mathrm{MHz}, \mathrm{CDCl}_{3}\right) \delta 51.1$ $\left(2 \mathrm{CH}_{2}\right), 66.9\left(2 \mathrm{CH}_{2}\right), 109.0(\mathrm{CH}), 124.0(\mathrm{CH}), 138.0(\mathrm{CH}), 138.6(\mathrm{C})$, $145.1(\mathrm{C}), 147.4(\mathrm{CH}), 151.5(\mathrm{CH}), 154.9$ (C). HRMS (EI) $\mathrm{m} / \mathrm{z}$ calcd for $\mathrm{C}_{12} \mathrm{H}_{13} \mathrm{~N}_{3} \mathrm{O}: 215.1059$; found: 215.1055 .

General procedure 3 for the preparation of 2-thienylzinc chloride or 2-benzothienylzinc chloride (insertion method A), ${ }^{[28]}$ and subsequent amination. ${ }^{[17]}$

A dry and argon flushed Schlenk flask equipped with a magnetic stirring bar and a septum was charged with magnesium turnings $(0.30 \mathrm{~g}$, $12.5 \mathrm{mmol})$, LiCl $(0.32 \mathrm{~g}, 7.5 \mathrm{mmol})$ and THF $(15 \mathrm{~mL})$. The aromatic bromide $(5.0 \mathrm{mmol})$ was added dropwise. If necessary, the Schlenk flask was placed in a water bath for cooling during the initial heat evolution of the insertion reaction. The progress of the insertion reaction was monitored by GC-analysis of reaction aliquots quenched with an aqueous saturated $\mathrm{NH}_{4} \mathrm{Cl}$ solution and/or iodine. Upon completion of the insertion, $\mathrm{ZnCl}_{2}(1.0 \mathrm{M})$ solution $(6.0 \mathrm{~mL}, 6.0 \mathrm{mmol})$ was added dropwise. The organozinc reagent was titrated using iodine solution (1.0 M in THF) before use in the amination reaction. A dry and argon flushed flask equipped with a magnetic stirring bar and a septum was charged with the $\mathrm{N}$-benzoyloxy secondary amine $(0.50 \mathrm{mmol})$ and THF $(2 \mathrm{~mL})$. To the mixture $\mathrm{CoCl}_{2} \cdot 2 \mathrm{LiCl}(50 \mu \mathrm{mol})$ was added as a $1.0 \mathrm{M}$ solution in THF. Then, the prepared organozinc chloride $(0.55 \mathrm{mmol})$ in THF was added dropwise. The solution was stirred for $2 \mathrm{~h}$ at room temperature and the reaction progress checked by thin layer chromatography. Upon completion of starting material (approximately less than $2 \mathrm{~h}$ ), the reaction was quenched with saturated aqueous $\mathrm{NH}_{4} \mathrm{Cl}$ solution $(1 \mathrm{~mL})$ and the product was extracted with AcOEt $(3 \times 15 \mathrm{~mL})$. The combined organic layers were washed with saturated aqueous $\mathrm{NaHCO}_{3}$ solution $(15 \mathrm{~mL})$ and brine $(15 \mathrm{~mL})$, dried over $\mathrm{MgSO}_{4}$, filtered and concentrated under reduced pressure. The crude product was purified by chromatography over silica gel (the eluent is given in the product description).

\section{2-(Morpholino)benzothiophene (1c).}

The general procedure 3 using 2-bromobenzothiophene $(1.1 \mathrm{~g})$ and $\mathrm{N}$ (benzoyloxy)morpholine $(0.10 \mathrm{~g})$ gave 1c (eluent: hexanes-AcOEt 90:10 in $96 \%$ yield $(0.10 \mathrm{~g})$ as a white powder: IR (ATR): $900,1012,1090$, $1263,1298,1374,1435,1526,1666,2838 \mathrm{~cm}^{-1} ;{ }^{1} \mathrm{H}$ NMR $(400 \mathrm{MHz}$, $\left.\mathrm{CDCl}_{3}\right) \delta 3.24(\mathrm{t}, 4 \mathrm{H}, J=4.9 \mathrm{~Hz}), 3.86(\mathrm{t}, 4 \mathrm{H}, J=4.8 \mathrm{~Hz}), 6.23(\mathrm{~s}, 1 \mathrm{H}, \mathrm{H} 3)$, $7.10(\mathrm{t}, 1 \mathrm{H}, J=7.6 \mathrm{~Hz}), 7.25(\mathrm{t}, 1 \mathrm{H}, J=7.4 \mathrm{~Hz}), 7.48(\mathrm{~d}, 1 \mathrm{H}, J=7.9 \mathrm{~Hz})$, $7.61(\mathrm{~d}, 1 \mathrm{H}, J=7.9 \mathrm{~Hz}) ;{ }^{13} \mathrm{C} \mathrm{NMR}\left(101 \mathrm{MHz}, \mathrm{CDCl}_{3}\right) \delta 51.1\left(2 \mathrm{CH}_{2}\right), 66.4$ $\left(2 \mathrm{CH}_{2}\right), 99.6(\mathrm{CH}), 121.2(\mathrm{CH}), 121.7(\mathrm{CH}), 121.8(\mathrm{CH}), 124.7(\mathrm{CH})$, 132.8 (C), 140.4 (C), 157.9 (C). These data are as reported previously. ${ }^{[10 a]} \mathrm{MS}(\mathrm{El}, 70 \mathrm{eV}) \mathrm{m} / \mathrm{z}$ (\%) 69 (12), 89 (44), 121 (17), 133 (14), 134 (72), 147 (50), 160 (100), 161 (99), 219 (19); HRMS (EI) m/z calcd for $\mathrm{C}_{12} \mathrm{H}_{13} \mathrm{NOS}$ : 219.0718; found: 219.0711 .

2-Morpholinothiophene (2c). 
The general procedure 3 using 2-bromothiophene $(0.82 \mathrm{~g})$ and $\mathrm{N}$ (benzoyloxy)morpholine $(0.10 \mathrm{~g})$ gave 2c (eluent: hexanes-AcOEt 90:10) in $80 \%$ yield $(68 \mathrm{mg})$ as a brown oil: IR (ATR): $928,1028,1090,1208$, 1269, 1293, 1374, 1443, 1466, 1528, $2853 \mathrm{~cm}^{-1}$; ${ }^{1} \mathrm{H}$ NMR $(400 \mathrm{MHz}$, $\left.\mathrm{CDCl}_{3}\right) \delta 3.12-3.14(\mathrm{~m}, 4 \mathrm{H}), 3.83-3.85(\mathrm{~m}, 4 \mathrm{H}), 6.16(\mathrm{dd}, 1 \mathrm{H}, J=3.7$ and $1.4 \mathrm{~Hz}, \mathrm{H} 3), 6.64(\mathrm{dd}, 1 \mathrm{H}, J=5.2$ and $1.1 \mathrm{~Hz}, \mathrm{H} 5), 6.80$ (dd, $1 \mathrm{H}, J=5.4$ and $3.8 \mathrm{~Hz}, \mathrm{H} 4) ;{ }^{13} \mathrm{C} \mathrm{NMR}\left(101 \mathrm{MHz}, \mathrm{CDCl}_{3}\right) \delta 52.2\left(2 \mathrm{CH}_{2}\right), 66.7\left(2 \mathrm{CH}_{2}\right)$, $105.9(\mathrm{CH}), 113.1(\mathrm{CH}), 126.4(\mathrm{CH}), 159.5(\mathrm{C})$. These data are as reported previously. ${ }^{[57]} \mathrm{MS}(\mathrm{El}, 70 \mathrm{eV}) \mathrm{m} / \mathrm{z}(\%) 110$ (33), 111 (61), 154 (11), 169 (100); HRMS (EI) $\mathrm{m} / z$ calcd for $\mathrm{C}_{8} \mathrm{H}_{11} \mathrm{NOS}$ : 169.0561; found: 169.0554 .

\section{2-[Di(2-methoxyethyl)amino]thiophene (2e).}

The general procedure 3 using 2-bromothiophene $(0.82 \mathrm{~g})$ and $N$ (benzoyloxy)di(2-methoxyethyl)amine $(0.13 \mathrm{~g})$ gave $2 \mathrm{e}$ (eluent: hexanesAcOEt 90:10) in $52 \%$ yield $(56 \mathrm{mg}$ ) as a brown oil: IR (ATR): 1012, 1100, $1185,1360,1446,1533,1639,2922 \mathrm{~cm}^{-1} ;{ }^{1} \mathrm{H}$ NMR $\left(400 \mathrm{MHz}, \mathrm{CDCl}_{3}\right) \delta$ 3.35 (s, $6 \mathrm{H}, \mathrm{OMe}$ ), 3.49 (td, $4 \mathrm{H}, J=5.8$ and $0.7 \mathrm{~Hz}, \mathrm{CH}_{2}$ ), 3.57 (td, $4 \mathrm{H}, J$ $=5.8$ and $\left.0.85 \mathrm{~Hz}, \mathrm{CH}_{2}\right), 5.90(\mathrm{dd}, 1 \mathrm{H}, J=3.7$ and $1.3 \mathrm{~Hz}, \mathrm{H} 3), 6.42(\mathrm{dd}$ $1 \mathrm{H}, J=5.4$ and $1.4 \mathrm{~Hz}, \mathrm{H} 5), 6.75(\mathrm{dd}, 1 \mathrm{H}, J=5.5$ and $3.7 \mathrm{~Hz}, \mathrm{H} 4) ;{ }^{13} \mathrm{C}$ $\operatorname{NMR}\left(101 \mathrm{MHz}, \mathrm{CDCl}_{3}\right) \delta 54.3\left(2 \mathrm{CH}_{2}\right), 59.1\left(2 \mathrm{CH}_{3}\right), 70.2\left(2 \mathrm{CH}_{2}\right), 102.3$ $(\mathrm{CH}), 109.3(\mathrm{CH}), 126.7(\mathrm{CH}), 157.3(\mathrm{C}) ; \mathrm{MS}(\mathrm{El}, 70 \mathrm{eV}) \mathrm{m} / \mathrm{z}(\%) 112$ (10), 138 (12), 170 (100), 215 (31); HRMS (EI) m/z calcd for $\mathrm{C}_{10} \mathrm{H}_{17} \mathrm{NO}_{2} \mathrm{~S}: 215.0980$; found: 215.0974 .

\section{2-[8-(1,4-Dioxa-8-azaspiro[4.5]decyl)]thiophene (2f).}

The general procedure 3 using 2-bromothiophene $(0.82 \mathrm{~g})$ and $\mathrm{N}$ (benzoyloxy)-1,4-dioxa-8-azaspiro[4.5]decane $(0.13 \mathrm{~g})$ gave $2 \mathrm{f}$ (eluent: hexanes-AcOEt $90: 10)$ in $78 \%$ yield $(88 \mathrm{mg})$ as a brown oil: IR (ATR): 1035, 1100, 1141, 1226, 1363, 1454, 1527, $2955 \mathrm{~cm}^{-1} ;{ }^{1} \mathrm{H}$ NMR (400 $\left.\mathrm{MHz}, \mathrm{CDCl}_{3}\right) \delta 1.85$ (t, $4 \mathrm{H}, J=5.9 \mathrm{~Hz}$ ), 3.28 (t, $4 \mathrm{H}, J=5.8 \mathrm{~Hz}$ ), 3.98 (s, $4 \mathrm{H}, \mathrm{OCH}_{2} \mathrm{CH}_{2} \mathrm{O}$ ), 6.12 (dd, $1 \mathrm{H}, J=3.8$ and $1.4 \mathrm{~Hz}, \mathrm{H} 3$ ), 6.59 (dd, $1 \mathrm{H}, J=$ 5.5 and $1.4 \mathrm{~Hz}, \mathrm{H} 5), 6.76(\mathrm{dd}, 1 \mathrm{H}, J=5.5$ and $3.8 \mathrm{~Hz}, \mathrm{H} 4) ;{ }^{13} \mathrm{C}$ NMR $(101$ $\left.\mathrm{MHz}, \mathrm{CDCl}_{3}\right) \delta 34.2\left(2 \mathrm{CH}_{2}\right), 50.5\left(2 \mathrm{CH}_{2}\right), 64.5\left(2 \mathrm{CH}_{2}\right), 106.1(\mathrm{CH}), 106.8$ (C, C5'), $112.5(\mathrm{CH}), 126.2(\mathrm{CH}), 159.1$ (C); MS (El, $70 \mathrm{eV}) \mathrm{m} / \mathrm{z}(\%) 110$ (25), 111 (67), 138 (21), 164 (13), 180 (12), 225 (100), 226 (11); HRMS (EI) $\mathrm{m} / \mathrm{z}$ calcd for $\mathrm{C}_{11} \mathrm{H}_{15} \mathrm{NO}_{2} \mathrm{~S}$ : 225.0823; found: 225.0816 .

General procedure 4 for the preparation of 2-thiazolylzinc chloride or 2-benzothiazolylzinc chloride (exchange method B), ${ }^{[29]}$ and subsequent amination. ${ }^{[17]}$

A dry and argon flushed $10 \mathrm{~mL}$ flask, equipped with a magnetic stirrer and a septum, was charged with iPrMgCl. $\mathrm{LiCl}(1.0 \mathrm{~mL}, 1.05 \mathrm{mmol}$, $1.05 \mathrm{M}$ in THF). The neat aryl bromide $(1.0 \mathrm{mmol})$ was added at room temperature. The reaction mixture was stirred for $0.5 \mathrm{~h}$, and the completion of the $\mathrm{Br} / \mathrm{Mg}$ exchange was checked by GC-analysis using tetradecane as internal standard. Upon completion of the exchange, $\mathrm{ZnCl}_{2}(1.0 \mathrm{M})$ solution $(1.2 \mathrm{~mL}, 1.2 \mathrm{mmol})$ was added dropwise. The organozinc reagent was titrated using iodine solution (1.0 M in THF) before use in the amination reaction. A dry and argon flushed flask equipped with a magnetic stirring bar and a septum was charged with the $\mathrm{N}$-benzoyloxy secondary amine $(0.50 \mathrm{mmol})$ and THF $(2 \mathrm{~mL})$. To the mixture $\mathrm{CoCl}_{2} \cdot 2 \mathrm{LiCl}(50 \mu \mathrm{mol})$ was added as a $1.0 \mathrm{M}$ solution in THF. Then, the prepared organozinc chloride $(0.55 \mathrm{mmol})$ in THF was added dropwise. The solution was stirred for $2 \mathrm{~h}$ at room temperature and the reaction progress checked by thin layer chromatography. Upon completion of starting material (approximately less than $2 \mathrm{~h}$ ), the reaction was quenched with saturated aqueous $\mathrm{NH}_{4} \mathrm{Cl}$ solution $(1 \mathrm{~mL})$ and the product was extracted with AcOEt $(3 \times 15 \mathrm{~mL})$. The combined organic layers were washed with saturated aqueous $\mathrm{NaHCO}_{3}$ solution $(15 \mathrm{~mL})$ and brine $(15 \mathrm{~mL})$, dried over $\mathrm{MgSO}_{4}$, filtered and concentrated under reduced pressure. The crude product was purified by chromatography over silica gel (the eluent is given in the product description).

\section{2-Morpholinothiazole (4c).}

The general procedure 4 using 2-bromothiazole $(0.16 \mathrm{~g})$ and $N$ (benzoyloxy)morpholine $(0.10 \mathrm{~g})$ gave 4c (eluent: hexanes-AcOEt 90:10) in $76 \%$ yield $(65 \mathrm{mg})$ as a brown oil: IR (ATR): $905,1034,1140,1229$, $1270,1373,1447,1511,2852 \mathrm{~cm}^{-1} ;{ }^{1} \mathrm{H}$ NMR $\left(400 \mathrm{MHz}, \mathrm{CDCl}_{3}\right) \delta 3.46-$ $3.48(\mathrm{~m}, 4 \mathrm{H}), 3.81-3.83(\mathrm{~m}, 4 \mathrm{H}), 6.60(\mathrm{~d}, 1 \mathrm{H}, J=3.6 \mathrm{~Hz}), 7.22(\mathrm{~d}, 1 \mathrm{H}, J=$ $3.7 \mathrm{~Hz}) ;{ }^{13} \mathrm{C}$ NMR $\left(101 \mathrm{MHz}, \mathrm{CDCl}_{3}\right) \delta 48.9\left(2 \mathrm{CH}_{2}\right), 66.1\left(2 \mathrm{CH}_{2}\right), 108.1$ $(\mathrm{CH}), 139.8(\mathrm{CH}), 172.6(\mathrm{C})$. These data are as reported previously. ${ }^{[58]}$ MS (El, $70 \mathrm{eV}) \mathrm{m} / \mathrm{z}(\%) 58$ (18), 85 (49), 86 (15), 99 (14), 100 (33), 101 (11), 111 (19), 112 (34), 113 (88), 125 (32), 126 (13), 139 (42), 151 (12), 155 (20), 168 (22), 169 (23), 170 (100); HRMS (EI) m/z calcd for $\mathrm{C}_{7} \mathrm{H}_{10} \mathrm{~N}_{2} \mathrm{OS}: 170.0514$; found: 170.0506 .

\section{2-[Di(2-methoxyethyl)amino]benzothiazole (5e).}

The general procedure 4 using 2-bromobenzothiazole $(0.21 \mathrm{~g})$ and $\mathrm{N}$ (benzoyloxy)di(2-methoxyethyl)amine $(0.13 \mathrm{~g})$ gave $5 \mathrm{e}$ (eluent: hexanesAcOEt $90: 10$ ) in $56 \%$ yield $(74 \mathrm{mg}$ ) as a yellow oil: IR (ATR): 749,1111 1309, 1442, 1549, 1560, 1702, $2875 \mathrm{~cm}^{-1} ;{ }^{1} \mathrm{H} \mathrm{NMR}\left(400 \mathrm{MHz}, \mathrm{CDCl}_{3}\right) \delta$ $3.40(\mathrm{~s}, 6 \mathrm{H}, \mathrm{OMe}), 3.70(\mathrm{t}, 4 \mathrm{H}, J=5.5 \mathrm{~Hz}), 3.82(\mathrm{t}, 4 \mathrm{H}, J=5.5 \mathrm{~Hz}), 7.04$ (ddd, $1 \mathrm{H}, J=7.8,7.3$ and $1.2 \mathrm{~Hz}$ ), 7.27 (ddd, $1 \mathrm{H}, J=8.6,7.4$ and $1.3 \mathrm{~Hz}$ ), 7.53 (ddd, $1 \mathrm{H}, J=8.1,1.0$ and $0.4 \mathrm{~Hz}$ ), 7.59-7.61 (ddd, $1 \mathrm{H}, J=7.8,1.2$ and $0.4 \mathrm{~Hz}) ;{ }^{13} \mathrm{C}$ NMR $\left(101 \mathrm{MHz}, \mathrm{CDCl}_{3}\right) \delta 52.3\left(2 \mathrm{CH}_{2}\right), 59.3\left(2 \mathrm{CH}_{3}\right)$, $70.8\left(2 \mathrm{CH}_{2}\right), 119.1(\mathrm{CH}), 120.9(\mathrm{CH}), 121.3(\mathrm{CH}), 126.2(\mathrm{CH}), 131.1(\mathrm{C})$, 153.4 (C), 168.2 (C). These data are as reported previously. ${ }^{[59]} \mathrm{MS}$ (EI, $70 \mathrm{eV}) \mathrm{m} / z$ (\%) 136 (16), 150 (21), 163 (100), 178 (25), 191 (43), 208 (36), 221 (20); HRMS (EI) $\mathrm{m} / z$ calcd for $\mathrm{C}_{13} \mathrm{H}_{18} \mathrm{~N}_{2} \mathrm{O}_{2} \mathrm{~S}: 266.1089$; found: 266.1079.

\section{2-[8-(1,4-Dioxa-8-azaspiro[4.5]decyl)]benzothiazole (5f).}

The general procedure 4 using 2-bromobenzothiazole $(0.21 \mathrm{~g})$ and $\mathrm{N}$ (benzoyloxy)-1,4-dioxa-8-azaspiro[4.5]decane $(0.13 \mathrm{~g})$ gave $\mathbf{5 f}$ (eluent: hexanes-AcOEt $90: 10)$ in $88 \%$ yield $(0.12 \mathrm{~g})$ as a yellow oil: IR (ATR) 922, 1099, 1291, 1446, 1543, 1595, $2925 \mathrm{~cm}^{-1} ;{ }^{1} \mathrm{H}$ NMR $(400 \mathrm{MHz}$, $\left.\mathrm{CDCl}_{3}\right) \delta 1.83(\mathrm{t}, 4 \mathrm{H}, J=5.9 \mathrm{~Hz}), 3.75(\mathrm{dd}, 4 \mathrm{H}, J=6.6$ and $5.1 \mathrm{~Hz}), 4.02$ (s, $4 \mathrm{H}, \mathrm{OCH}_{2} \mathrm{CH}_{2} \mathrm{O}$ ), 7.06 (td, $1 \mathrm{H}, J=7.6$ and $1.1 \mathrm{~Hz}$ ), 7.28 (ddd, $1 \mathrm{H}, J=$ $8.5,7.4$ and $1.3 \mathrm{~Hz}), 7.54(\mathrm{dd}, 1 \mathrm{H}, J=8.1$ and $0.6 \mathrm{~Hz}), 7.58-7.60(\mathrm{~m}$ $1 \mathrm{H}) ;{ }^{13} \mathrm{C} \mathrm{NMR}\left(101 \mathrm{MHz}, \mathrm{CDCl}_{3}\right) \delta 34.7\left(2 \mathrm{CH}_{2}\right), 47.1\left(2 \mathrm{CH}_{2}\right), 64.8\left(2 \mathrm{CH}_{2}\right)$, $107.2\left(\mathrm{C}, \mathrm{C}^{\prime}\right), 119.3(\mathrm{CH}), 121.0(\mathrm{CH}), 121.7(\mathrm{CH}), 126.3(\mathrm{CH}), 131.3$ (C), $153.2(\mathrm{C}), 168.6(\mathrm{C})$. These data are as reported previously. ${ }^{[60]} \mathrm{MS}$ (El, $70 \mathrm{eV}) \mathrm{m} / \mathrm{z}(\%) 99$ (20), 108 (14), 134 (12), 135 (46), 136 (12), 149 (59), 161 (13), 162 (13), 163 (77), 175 (89), 176 (39), 177 (25), 189 (12), 203 (12), 207 (16), 276 (100); HRMS (EI) $m / z$ calcd for $\mathrm{C}_{14} \mathrm{H}_{16} \mathrm{~N}_{2} \mathrm{O}_{2} \mathrm{~S}$ : 276.0932; found: 276.0922

\section{2-(1-Azepanyl)benzothiazole (5g).}

The general procedure 4 using 2-bromobenzothiazole $(0.21 \mathrm{~g})$ and $\mathrm{N}$ (benzoyloxy)azepane $(0.11 \mathrm{~g})$ gave $\mathbf{5 g}$ (eluent: hexanes-AcOEt 90:10) in $89 \%$ yield $(0.10 \mathrm{~g})$ as a yellow oil: IR (ATR): $748,1249,1299,1359,1441$, 1560, $2923 \mathrm{~cm}^{-1} ;{ }^{1} \mathrm{H}$ NMR $\left(400 \mathrm{MHz}, \mathrm{CDCl}_{3}\right) \delta 1.61-1.64(\mathrm{~m}, 4 \mathrm{H}), 1.85-$ $1.91(\mathrm{~m}, 4 \mathrm{H}), 3.69(\mathrm{t}, 4 \mathrm{H}, J=6.0 \mathrm{~Hz}), 7.03(\mathrm{td}, 1 \mathrm{H}, J=7.6$ and $1.1 \mathrm{~Hz}$ ), 7.28 (ddd, $1 \mathrm{H}, J=8.2,7.4$ and $1.3 \mathrm{~Hz}$ ), 7.55 (ddd, $1 \mathrm{H}, J=8.0,1.0$ and $0.4 \mathrm{~Hz}$ ), 7.59 (ddd, $1 \mathrm{H}, J=7.8,1.2$ and $0.4 \mathrm{~Hz})$ ); ${ }^{13} \mathrm{C}$ NMR $(101 \mathrm{MHz}$, $\left.\mathrm{CDCl}_{3}\right) \delta 27.9\left(2 \mathrm{CH}_{2}\right), 28.2\left(2 \mathrm{CH}_{2}\right), 51.1\left(2 \mathrm{CH}_{2}\right), 118.7(\mathrm{CH}), 120.7(\mathrm{CH})$, $120.9(\mathrm{CH}), 126.2(\mathrm{CH}), 130.9(\mathrm{C}), 153.7(\mathrm{C}), 168.5(\mathrm{C})$. These data are as reported previously. ${ }^{[61]} \mathrm{MS}(\mathrm{El}, 70 \mathrm{eV}) \mathrm{m} / \mathrm{z}(\%) 98(11), 135$ (17), 136 
(31), 149 (38), 150 (49), 162 (23), 163 (46), 175 (37), 177 (14), 189 (65), 199 (23), 203 (18), 217 (27), 232 (100), 233 (14); HRMS (EI) $\mathrm{m} / z$ calcd for $\mathrm{C}_{13} \mathrm{H}_{16} \mathrm{~N}_{2} \mathrm{~S}: 232.1034$; found: 232.1030 .

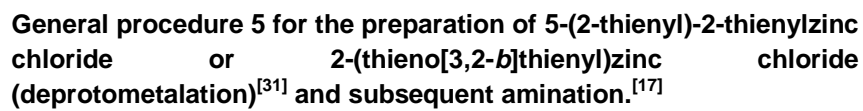

A dry and argon flushed Schlenk flask, equipped with a magnetic stirrer and a septum, was charged with the starting bithiophene or thienothiophene $(0.50 \mathrm{mmol})$ in THF (approximately $1.0 \mathrm{M}$ solution) at room temperature. A solution of TMPMgCl. $\mathrm{LiCl}(0.55 \mathrm{mmol})$ in THF was added dropwise and the reaction mixture stirred for $1 \mathrm{~h}$ (the completion of the reaction was checked by GC analysis of reaction aliquots quenched with a solution of $\mathrm{I}_{2}$ in THF). Upon completion of the deprotonation, $\mathrm{ZnCl}_{2}$ $(1.0 \mathrm{M})$ solution $(0.60 \mathrm{ml}, 0.60 \mathrm{mmol})$ was added dropwise. The organozinc reagent was titrated using iodine solution (1.0 M in THF) before use in the amination reaction. A dry and argon flushed flask equipped with a magnetic stirring bar and a septum was charged with the $\mathrm{N}$-benzoyloxy secondary amine $(0.50 \mathrm{mmol})$ and THF (about $2 \mathrm{~mL}$ ). $\mathrm{CoCl}_{2} \cdot 2 \mathrm{LiCl}(50 \mu \mathrm{mol} ; 1.0 \mathrm{M}$ solution in THF) and TMEDA $(15 \mu \mathrm{L}$, $0.10 \mathrm{mmol}$ ) were added to the mixture. Then, the prepared organozinc chloride $(0.55 \mathrm{mmol})$ in THF was added dropwise. The solution was stirred for $2 \mathrm{~h}$ at room temperature and the reaction progress checked by thin layer chromatography. Upon completion of starting material (approximately less than $2 \mathrm{~h}$ ), the reaction was quenched with saturated aqueous $\mathrm{NH}_{4} \mathrm{Cl}$ solution $(1 \mathrm{~mL})$ and the product was extracted with AcOEt $(3 \times 15 \mathrm{~mL})$. The combined organic layers were washed with saturated aqueous $\mathrm{NaHCO}_{3}$ solution $(15 \mathrm{~mL})$ and brine $(15 \mathrm{~mL})$, dried over $\mathrm{MgSO}_{4}$, filtered and concentrated under reduced pressure. The crude product was purified by chromatography over silica gel (the eluent is given in the product description).

\section{5-Morpholino-2,2'-bithiophene (6c)}

The general procedure 5, but without TMEDA, using 2,2'-bithiophene $(83 \mathrm{mg})$ and $\mathrm{N}$-(benzoyloxy)morpholine $(0.10 \mathrm{~g})$ gave $6 \mathrm{c}$ (eluent: hexanes-AcOEt $90: 10)$ in $72 \%$ yield $(90 \mathrm{mg})$ as a yellow powder: IR (ATR): 899, 1115, 1296, 1450, 1535, 1594, $2925 \mathrm{~cm}^{-1} ;{ }^{1} \mathrm{H}$ NMR (400 $\left.\mathrm{MHz}, \mathrm{CDCl}_{3}\right) \delta 3.15-3.16(\mathrm{~m}, 4 \mathrm{H}), 3.85-3.87(\mathrm{~m}, 4 \mathrm{H}), 6.08(\mathrm{~d}, 1 \mathrm{H}, J=3.9$ $\mathrm{Hz}), 6.88(\mathrm{~d}, 1 \mathrm{H}, J=3.7 \mathrm{~Hz}), 6.96\left(\mathrm{dd}, 1 \mathrm{H}, J=5.1\right.$ and $\left.3.6 \mathrm{~Hz}, \mathrm{H} 4^{\prime}\right), 6.99$ (dd, $1 \mathrm{H}, J=3.6$ and $1.2 \mathrm{~Hz}, \mathrm{H} 3^{\prime}$ ), 7.11 (dd, $1 \mathrm{H}, J=5.0$ and $1.3 \mathrm{~Hz}, \mathrm{H}^{\prime}$ ); ${ }^{13} \mathrm{C}$ NMR $\left(101 \mathrm{MHz}, \mathrm{CDCl}_{3}\right) \delta 51.6\left(2 \mathrm{CH}_{2}\right), 66.6\left(2 \mathrm{CH}_{2}\right), 106.0(\mathrm{CH})$, $122.1(\mathrm{CH}), 123.1(\mathrm{CH}), 123.2(\mathrm{CH}), 124.6(\mathrm{C}), 127.9(\mathrm{CH}), 138.5(\mathrm{C})$, 158.5 (C). These data are as reported previously. ${ }^{[62]} \mathrm{MS}(\mathrm{El}, 70 \mathrm{eV}) \mathrm{m} / \mathrm{z}$ (\%) 121 (16), 179 (12), 191 (38), 193 (77), 251 (100); HRMS (EI) m/z calcd for $\mathrm{C}_{12} \mathrm{H}_{13} \mathrm{NOS}_{2}$ : 251.0439; found: 251.0435.

\section{2-(Morpholino)thieno[3,2-b]thiophene (7c).}

The general procedure 5 using thieno[3,2-b]thiophene $(70 \mathrm{mg})$ and $\mathrm{N}$ (benzoyloxy)morpholine $(0.10 \mathrm{~g})$ gave $7 \mathrm{c}$ (eluent: hexanes- $\left.\mathrm{CH}_{2} \mathrm{Cl}_{2} 80: 20\right)$ in $92 \%$ yield $(0.10 \mathrm{~g})$ as a white powder: IR (ATR): $925,1100,1217$, $1261,1374,1442,1509,2831 \mathrm{~cm}^{-1} ;{ }^{1} \mathrm{H}$ NMR $\left(400 \mathrm{MHz}, \mathrm{CDCl}_{3}\right) \delta 3.16-$ $3.18(\mathrm{~m}, 4 \mathrm{H}), 3.85-3.88(\mathrm{~m}, 4 \mathrm{H}), 6.37(\mathrm{~s}, 1 \mathrm{H}), 7.10(\mathrm{~d}, 1 \mathrm{H}, J=5.2 \mathrm{~Hz})$, $7.12(\mathrm{~d}, 1 \mathrm{H}, J=5.2 \mathrm{~Hz}) ;{ }^{13} \mathrm{C} \mathrm{NMR}\left(101 \mathrm{MHz}, \mathrm{CDCl}_{3}\right) \delta 52.1\left(2 \mathrm{CH}_{2}\right), 66.4$ $\left(2 \mathrm{CH}_{2}\right), 99.5(\mathrm{CH}), 119.6(\mathrm{CH}), 122.8(\mathrm{CH}), 128.6(\mathrm{C}), 138.1(\mathrm{C}), 160.1$ (C). MS (El, $70 \mathrm{eV}) \mathrm{m} / \mathrm{z}(\%) 69$ (10), 122 (12), 139 (10), 140 (46), 153 (23), 166 (47), 167 (99), 207 (13), 223 (16), 225 (100); HRMS (EI) m/z calcd for $\mathrm{C}_{10} \mathrm{H}_{11} \mathrm{NOS}_{2}$ : 225.0282; found: 225.0276.

\section{2-[8-(1,4-Dioxa-8-azaspiro[4.5]decyl)]thieno[3,2-b]thiophene (7f).}

The general procedure 5 using thieno[3,2-b]thiophene $(70 \mathrm{mg})$ and $\mathrm{N}$ (benzoyloxy)-1,4-dioxa-8-azaspiro[4.5]decane $(0.13 \mathrm{~g})$ gave $7 \mathrm{f}$ (eluent: hexanes $\left.-\mathrm{CH}_{2} \mathrm{Cl}_{2} 80: 20\right)$ in $85 \%$ yield $(0.12 \mathrm{~g})$ as a green powder: IR (ATR): 1066, 1115, 1297, 1442, 1520, $2926 \mathrm{~cm}^{-1} ;{ }^{1} \mathrm{H}$ NMR $(400 \mathrm{MHz}$, $\left.\mathrm{CDCl}_{3}\right) \delta 1.87(\mathrm{t}, 4 \mathrm{H}, J=5.9 \mathrm{~Hz}$ ), $3.32(\mathrm{t}, 4 \mathrm{H}, J=5.8 \mathrm{~Hz}), 4.00(\mathrm{~s}, 4 \mathrm{H}$, $\left.\mathrm{OCH}_{2} \mathrm{CH}_{2} \mathrm{O}\right), 6.32(\mathrm{~s}, 1 \mathrm{H}), 7.08(\mathrm{~s}, 2 \mathrm{H}) ;{ }^{13} \mathrm{C}$ NMR $\left(101 \mathrm{MHz}, \mathrm{CDCl}_{3}\right) \delta$ $34.2\left(2 \mathrm{CH}_{2}\right), 50.4\left(2 \mathrm{CH}_{2}\right), 64.6\left(2 \mathrm{CH}_{2}\right), 98.9(\mathrm{CH}), 106.8(\mathrm{C}, \mathrm{C} 5), 119.6$ (CH), $121.9(\mathrm{CH}), 128.2$ (C), 138.4 (C), 160.7 (C); MS (El, $70 \mathrm{eV}) \mathrm{m} / \mathrm{z}$ (\%) 140 (22), 166 (20), 167 (100), 207 (38), 209 (21), 225 (48), 281 (34); HRMS (EI) $\mathrm{m} / z$ calcd for $\mathrm{C}_{13} \mathrm{H}_{15} \mathrm{NO}_{2} \mathrm{~S}_{2}: 281.0544$; found: 281.0537

\section{2-[2-(1,2,3,4-tetrahydroisoquinolyl)]thieno[3,2-b]thiophene (7h).}

The general procedure 5 using thieno[3,2-b]thiophene $(70 \mathrm{mg})$ and $\mathrm{N}$ (benzoyloxy)-1,2,3,4-tetrahydroisoquinoline $(0.13 \mathrm{~g})$ gave $\mathbf{7 h}$ (eluent: hexanes $\left.-\mathrm{CH}_{2} \mathrm{Cl}_{2} 80: 20\right)$ in $88 \%$ yield $(0.12 \mathrm{~g})$ as a green powder: IR (ATR): 928, 1196, 1380, 1454, 1519, $2831 \mathrm{~cm}^{-1} ;{ }^{1} \mathrm{H}$ NMR $(400 \mathrm{MHz}$ $\left.\mathrm{CDCl}_{3}\right) \delta 3.04(\mathrm{t}, 2 \mathrm{H}, J=6.0 \mathrm{~Hz}), 3.54(\mathrm{t}, 2 \mathrm{H}, J=6.0 \mathrm{~Hz}), 4.41(\mathrm{~s}, 2 \mathrm{H}$, $\mathrm{H}^{\prime}$ ), $6.40(\mathrm{~s}, 1 \mathrm{H}), 7.08(\mathrm{~d}, 1 \mathrm{H}, J=5.2 \mathrm{~Hz}), 7.11(\mathrm{~d}, 1 \mathrm{H}, J=5.2 \mathrm{~Hz}), 7.14$ $7.23(\mathrm{~m}, 4 \mathrm{H}) ;{ }^{13} \mathrm{C}$ NMR $\left(101 \mathrm{MHz}, \mathrm{CDCl}_{3}\right) \delta 28.8\left(\mathrm{CH}_{2}\right), 49.6\left(\mathrm{CH}_{2}\right), 53.1$ $\left(\mathrm{CH}_{2}\right), 97.9(\mathrm{CH}), 119.6(\mathrm{CH}), 121.8(\mathrm{CH}), 126.3(\mathrm{CH}), 126.5(\mathrm{CH}), 126.7$ $(\mathrm{CH}), 127.6(\mathrm{C}), 128.9(\mathrm{CH}), 133.1(\mathrm{C}), 134.0(\mathrm{C}), 138.6(\mathrm{C}), 160.1(\mathrm{C})$; MS (El, $70 \mathrm{eV}) \mathrm{m} / \mathrm{z}(\%) 73$ (13), 77 (12), 78 (15), 88 (11), 91 (16), 103 (16), 104 (58), 115 (24), 117 (49), 139 (17), 166 (68), 270 (30), 271 (100), 272 (21), 273 (13), 277 (22); HRMS (EI) $\mathrm{m} / \mathrm{z}$ calcd for $\mathrm{C}_{15} \mathrm{H}_{13} \mathrm{NS}_{2}$ : 271.0489; found: 271.0498. This compound is quite instable in $\mathrm{CDCl}_{3}$.

General procedure 6 for the preparation of 2-thiooxo-4-(1,3dithiolyl)zinc chloride (deprotometalation), ${ }^{[32]}$ and subsequent amination. ${ }^{[17]}$

A dry and argon flushed Schlenk flask was charged with a solution of 1,3dithiole-2-thione $(0.50 \mathrm{mmol})$ in anhydrous THF (approximately $0.5 \mathrm{M}$ solution). A solution of TMPMgCl. $\mathrm{LiCl}(0.55 \mathrm{mmol})$ in THF was added dropwise at $-78^{\circ} \mathrm{C}$ and the mixture was stirred at this temperature for $0.5 \mathrm{~h}$. The completion of the reaction was checked by GC analysis of reaction aliquots quenched with $\mathrm{I}_{2}$. Upon completion of the deprotonation, a $\mathrm{ZnCl}_{2}(0.60 \mathrm{~mL}, 0.60 \mathrm{mmol})$ solution $(1.0 \mathrm{M}$ in THF) was added dropwise. The organozinc reagent was titrated using iodine solution (1.0 M in THF) before use in the amination reaction. A dry and argon flushed flask equipped with a magnetic stirring bar and a septum was charged with the $\mathrm{N}$-benzoyloxy secondary amine $(0.50 \mathrm{mmol})$ and THF (about $2 \mathrm{~mL}$ ). $\mathrm{CoCl}_{2} \cdot 2 \mathrm{LiCl}(50 \mu \mathrm{mol} ; 1.0 \mathrm{M}$ solution in THF) and TMEDA $(15 \mu \mathrm{L}, 0.10 \mathrm{mmol})$ were added to the mixture. Then, the prepared organozinc chloride $(0.55 \mathrm{mmol})$ in THF was added dropwise. The solution was stirred for $2 \mathrm{~h}$ at room temperature and the reaction progress checked by thin layer chromatography. Upon completion of starting material (approximately less than $2 \mathrm{~h}$ ), the reaction was quenched with saturated aqueous $\mathrm{NH}_{4} \mathrm{Cl}$ solution $(1 \mathrm{~mL})$ and the product was extracted with AcOEt $(3 \times 15 \mathrm{~mL})$. The combined organic layers were washed with saturated aqueous $\mathrm{NaHCO}_{3}$ solution $(15 \mathrm{~mL})$ and brine $(15 \mathrm{~mL})$, dried over $\mathrm{MgSO}_{4}$, filtered and concentrated under reduced pressure. The crude product was purified by chromatography over silica gel (the eluent is given in the product description).

\section{4-Morpholino-1,3-dithiole-2-thione (8c).}

The general procedure 6 using $N$-(benzoyloxy)morpholine $(0.10 \mathrm{~g})$ gave 8c (eluent: hexanes-AcOEt $90: 10)$ in $73 \%$ yield $(80 \mathrm{mg})$ as a yellow powder: IR (ATR): 867, 1000, 1111, 1214, 1273, 1375, 1442, 1531, 2834 $\mathrm{cm}^{-1} ;{ }^{1} \mathrm{H}$ NMR $\left(400 \mathrm{MHz}, \mathrm{CDCl}_{3}\right) \delta 3.07-3.10(\mathrm{~m}, 4 \mathrm{H}), 3.79-3.81(\mathrm{~m}, 4 \mathrm{H})$, $5.63(\mathrm{~s}, 1 \mathrm{H}, \mathrm{H} 5) ;{ }^{13} \mathrm{C} \mathrm{NMR}\left(101 \mathrm{MHz}, \mathrm{CDCl}_{3}\right) \delta 51.0\left(2 \mathrm{CH}_{2}\right), 66.1\left(2 \mathrm{CH}_{2}\right)$, 98.8 (CH, C5), 155.2 (C, C4), 207.9 (C, C=S); MS (El, $70 \mathrm{eV}) \mathrm{m} / \mathrm{z}(\%) 48$ 
(27), 49 (100), 50 (12), 52 (25), 60 (15), 73 (11), 75 (14), 84 (30), 115 (11), 143 (31); HRMS (EI) $\mathrm{m} / z$ calcd for $\mathrm{C}_{7} \mathrm{H}_{9} \mathrm{NOS}_{3}$ : 219.9846; found: 219.9902 .

\section{4-[Di(2-methoxyethyl)amino]-1,3-dithiole-2-thione (8e).}

The general procedure 6 using $N$-(benzoyloxy)di(2-methoxyethyl)amine $(0.13 \mathrm{~g})$ gave $8 \mathrm{e}$ (eluent: hexanes-AcOEt $90: 10)$ in $95 \%$ yield $(0.13 \mathrm{~g})$ as a red oil: IR (ATR): 942, 1054, 1099, 1363, 1455, 1534, $2978 \mathrm{~cm}^{-1} ;{ }^{1} \mathrm{H}$ NMR (400 MHz, $\left.\mathrm{CDCl}_{3}\right) \delta 3.35(\mathrm{~s}, 6 \mathrm{H}, \mathrm{OMe}), 3.43(\mathrm{t}, 4 \mathrm{H}, J=6.0 \mathrm{~Hz})$, $3.54(\mathrm{t}, 4 \mathrm{H}, J=6.0 \mathrm{~Hz}), 5.39(\mathrm{~s}, 1 \mathrm{H}) ;{ }^{13} \mathrm{C} \mathrm{NMR}\left(101 \mathrm{MHz}, \mathrm{CDCl}_{3}\right) \delta 53.9$ $\left(2 \mathrm{CH}_{2}\right), 59.2\left(2 \mathrm{CH}_{3}\right), 70.1\left(2 \mathrm{CH}_{2}\right), 93.1(\mathrm{CH}, \mathrm{C} 5), 153.6(\mathrm{C}, \mathrm{C} 4), 206.7(\mathrm{C}$ $\mathrm{C}=\mathrm{S}$ ); MS (El, $70 \mathrm{eV}$ ) m/z (\%) 76 (100), 78 (10), 207 (13), 225 (11); HRMS (EI) $\mathrm{m} / z$ calcd for $\mathrm{C}_{9} \mathrm{H}_{15} \mathrm{NO}_{2} \mathrm{~S}_{3}$ : 265.0265; found: 265.0264 .

\section{4-[8-(1,4-Dioxa-8-azaspiro[4.5]decyl)]-1,3-dithiole-2-thione (8f).}

The general procedure 6 using $N$-(benzoyloxy)-1,4-dioxa-8azaspiro[4.5]decane $(0.13 \mathrm{~g})$ gave $8 \mathrm{f}$ (eluent: hexanes-AcOEt 90:10) in $98 \%$ yield $(0.13 \mathrm{~g})$ as a yellow powder: IR (ATR): $920,1022,1099,1145$,

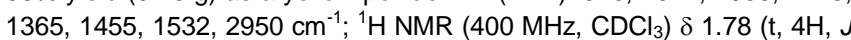
$\left.=5.9 \mathrm{~Hz}, 2 \mathrm{CH}_{2}\right), 3.20\left(\mathrm{t}, 4 \mathrm{H}, \mathrm{J}=5.9 \mathrm{~Hz}, 2 \mathrm{CH}_{2}\right), 3.94\left(\mathrm{~s}, 4 \mathrm{H}, \mathrm{OCH}_{2} \mathrm{CH}_{2} \mathrm{O}\right)$, $5.55(\mathrm{~s}, 1 \mathrm{H}, \mathrm{H} 5) ;{ }^{13} \mathrm{C}$ NMR $\left(101 \mathrm{MHz}, \mathrm{CDCl}_{3}\right) \delta 33.9\left(2 \mathrm{CH}_{2}\right), 49.5\left(2 \mathrm{CH}_{2}\right)$, $64.6\left(2 \mathrm{CH}_{2}\right), 98.5(\mathrm{CH}, \mathrm{C} 5), 106.0$ (C, C5'), 155.1 (C, C4), 208.2 (C, $\mathrm{C}=\mathrm{S}$ ); MS (El, 70 eV) m/z (\%) 43 (100), 45 (14), 61 (19), 70 (14), 98 (13) 275 (50); HRMS (EI) $\mathrm{m} / z$ calcd for $\mathrm{C}_{10} \mathrm{H}_{13} \mathrm{NO}_{2} \mathrm{~S}_{3}$ : 275.0108; found: 275.0100 .

\section{4-[2-(1,2,3,4-tetrahydroisoquinolyl)]-1,3-dithiole-2-thione (8h).}

The general procedure 6 using $N$-(benzoyloxy)-1,2,3,4tetrahydroisoquinoline $(0.13 \mathrm{~g})$ gave $8 \mathrm{~h}$ (eluent: hexanes-AcOEt $90: 10)$ in $96 \%$ yield $(0.13 \mathrm{~g})$ as a yellow powder: IR (ATR): $842,1038,1179,1381$, 1448, 1532, $2921 \mathrm{~cm}^{-1} ;{ }^{1} \mathrm{H}$ NMR $\left(400 \mathrm{MHz}, \mathrm{CDCl}_{3}\right) \delta 2.98(\mathrm{t}, 2 \mathrm{H}, J=5.5$ $\mathrm{Hz}$ ), 3.46 (t, $2 \mathrm{H}, J=6.0 \mathrm{~Hz}$ ), 4.33 (s, 2H, H1'), 5.57 (s, 1H, H5), 7.11 (dd, $1 \mathrm{H}, J=5.2$ and $3.6 \mathrm{~Hz}), 7.16(\mathrm{dd}, 1 \mathrm{H}, J=5.3$ and $3.8 \mathrm{~Hz}), 7.20-7.24(\mathrm{~m}$, $2 \mathrm{H}) ;{ }^{13} \mathrm{C}$ NMR $\left(101 \mathrm{MHz}, \mathrm{CDCl}_{3}\right) \delta 28.6\left(\mathrm{CH}_{2}\right), 49.1\left(\mathrm{CH}_{2}\right), 52.0\left(\mathrm{CH}_{2}\right)$, $96.3(\mathrm{CH}, \mathrm{C5}), 126.4(\mathrm{CH}), 126.7(\mathrm{CH}), 127.2(\mathrm{CH}), 128.9(\mathrm{CH}), 131.9$ (C), 133.5 (C), 154.3 (C, C4), 207.5 (C, C=S); MS (El, 70 eV) m/z (\%) 42 (42), 75 (11), 91 (16), 103 (12), 104 (15), 115 (24), 117 (81), 118 (18), 132 (14), 156 (21), 188 (57), 189 (15), 265 (100), 267 (14); HRMS (El) $\mathrm{m} / \mathrm{z}$ calcd for $\mathrm{C}_{12} \mathrm{H}_{11} \mathrm{NS}_{3}: 265.0054$; found: 265.0048 .

\section{Methyl 3-(phenylamino)-2-thiophenecarboxylate (9a).}

To iodobenzene $(0.11 \mathrm{~mL}, 1.0 \mathrm{mmol})$ in $\mathrm{Bu}_{2} \mathrm{O}(1.5 \mathrm{~mL})$ were successively added activated $\mathrm{Cu}(13 \mathrm{mg}, 0.20 \mathrm{mmol})$, methyl 3-amino-2thiophenecarboxylate $(0.16 \mathrm{~g}, 1.0 \mathrm{mmol})$ and $\mathrm{K}_{2} \mathrm{CO}_{3}(0.28 \mathrm{~g}, 2.0 \mathrm{mmol})$. The mixture was degassed and heated at $140{ }^{\circ} \mathrm{C}$ under argon for $24 \mathrm{~h}$. During this time, activated $\mathrm{Cu}(4 \times 13 \mathrm{mg}, 4 \times 0.20 \mathrm{mmol})$ was added after 2, 4, 6 and $8 \mathrm{~h}$ of heating. After cooling to room temperature, the mixture was concentrated. Addition of $\mathrm{H}_{2} \mathrm{O}(25 \mathrm{~mL})$, extraction with AcOEt $(3 \times 10 \mathrm{~mL})$, drying over $\mathrm{Na}_{2} \mathrm{SO}_{4}$, removal of the solvent and purification by chromatography on silica gel (eluent: hexanes-AcOEt 95:5; $\left.R_{f}=0.525\right)$ afforded $5 a$ in $79 \%$ yield $(0.18 \mathrm{~g})$ as a yellow powder: $\mathrm{mp} 68^{\circ} \mathrm{C}$ (lit. ${ }^{[35 \mathrm{a}]} 66-67^{\circ} \mathrm{C}$ ); IR (ATR): 692, 754, 772, 1090, 1226, 1254, 1394, 1418, 1435, 1566, 1593, 1667, 2948, $3309 \mathrm{~cm}^{-1} ;{ }^{1} \mathrm{H}$ NMR (300 $\left.\mathrm{MHz} \mathrm{CDCl}_{3}\right) \delta 3.88(\mathrm{~s}, 3 \mathrm{H}, \mathrm{OMe}), 7.06(\mathrm{t}, 1 \mathrm{H}, J=7.4 \mathrm{~Hz}, \mathrm{H} 4$ ), 7.10 (d, $1 \mathrm{H}, J=5.5 \mathrm{~Hz}, \mathrm{H} 4), 7.18\left(\mathrm{~d}, 2 \mathrm{H}, J=7.7 \mathrm{~Hz}, \mathrm{H} 2^{\prime}\right.$ and $\left.\mathrm{H} 66^{\prime}\right), 7.33$ (t, $2 \mathrm{H}, J=$ $7.7 \mathrm{~Hz}, \mathrm{H} 3$ ' and H5'), 7.35 (d, $1 \mathrm{H}, J=5.3 \mathrm{~Hz}, \mathrm{H} 5), 8.80$ (br s, $1 \mathrm{H}, \mathrm{NH}$ ); ${ }^{13} \mathrm{C}$ NMR $\left(75 \mathrm{MHz}, \mathrm{CDCl}_{3}\right) \delta 51.5\left(\mathrm{CH}_{3}\right), 103.0(\mathrm{C}, \mathrm{C} 2), 118.0(\mathrm{CH})$, $120.3\left(2 \mathrm{CH}, \mathrm{C} 2\right.$ ' and $\left.\mathrm{C6}^{\prime}\right), 123.1(\mathrm{CH}), 129.4\left(2 \mathrm{CH}, \mathrm{C}^{\prime}\right.$ and $\left.\mathrm{C}^{\prime}\right), 131.8$
$(\mathrm{CH}, \mathrm{C} 4), 141.5\left(\mathrm{C}, \mathrm{C} 1^{\prime}\right), 151.5(\mathrm{C}, \mathrm{C} 3), 165.2(\mathrm{C}, \mathrm{C}=\mathrm{O})$. These data are similar to those described previously. ${ }^{[35 a]}$

General procedure 7 for the bis- $\mathrm{N}$-arylation of methyl 3-amino-2thiophenecarboxylate using the same iodide.

To the required iodide $(2.0 \mathrm{mmol})$ in $\mathrm{Bu}_{2} \mathrm{O}(1.5 \mathrm{~mL})$ were successively added activated $\mathrm{Cu}(13 \mathrm{mg}, 0.20 \mathrm{mmol})$, methyl 3-amino-2thiophenecarboxylate $(0.16 \mathrm{~g}, 1.0 \mathrm{mmol})$ and $\mathrm{K}_{2} \mathrm{CO}_{3}(0.28 \mathrm{~g}, 2.0 \mathrm{mmol})$. The mixture was degassed and heated at $140{ }^{\circ} \mathrm{C}$ under argon for $24 \mathrm{~h}$. During this time, activated $\mathrm{Cu}(4 \times 13 \mathrm{mg}, 4 \times 0.20 \mathrm{mmol})$ was added after 2, 4, 6 and $8 \mathrm{~h}$ of heating. After cooling to room temperature, the mixture was concentrated. Addition of $\mathrm{H}_{2} \mathrm{O}(25 \mathrm{~mL})$, extraction with AcOEt $(3 \times 10 \mathrm{~mL})$, drying over $\mathrm{Na}_{2} \mathrm{SO}_{4}$, removal of the solvent and purification by chromatography on silica gel (the eluent is given in the product description) led to the expected compound.

\section{Methyl 3-(diphenylamino)-2-thiophenecarboxylate (10a).}

The general procedure 7 using iodobenzene $(0.22 \mathrm{~mL})$ gave $10 \mathrm{a}$ (eluent: hexanes-AcOEt $\left.95: 5 ; R_{\mathrm{f}}=0.35\right)$ in $88 \%$ yield $(0.27 \mathrm{~g})$ as a yellow oil; IR (ATR): 503, 583, 637, 674, 694, 754, 776, 853, 951, 1041, 1074, 1094, $1175,1226,1253,1394,1418,1438,1481,1524,1567,1592,1667$ 1714, $2950 \mathrm{~cm}^{-1}$; ${ }^{1} \mathrm{H}$ NMR (300 MHz, $\mathrm{CDCl}_{3}$ ) $\delta 3.53$ (s, 3H, OMe), 6.81 (d, $1 \mathrm{H}, J=5.3 \mathrm{~Hz}, \mathrm{H} 4), 7.00$ (t, 2H, J=7.2 Hz, H4'), $7.04(\mathrm{~d}, 4 \mathrm{H}, J=8.1 \mathrm{~Hz}$, $\mathrm{H} 2^{\prime}$ and $\left.\mathrm{H} 6^{\prime}\right), 7.23$ (t, $4 \mathrm{H}, J=7.8 \mathrm{~Hz}, \mathrm{H} 3^{\prime}$ and $\mathrm{H}^{\prime}$ ), $7.42(\mathrm{~d}, 1 \mathrm{H}, J=5.3$ $\mathrm{Hz}, \mathrm{H} 5) ;{ }^{13} \mathrm{C}$ NMR $\left(75 \mathrm{MHz}, \mathrm{CDCl}_{3}\right) \delta 51.7\left(\mathrm{CH}_{3}\right), 121.1(\mathrm{C}, \mathrm{C} 2), 122.7$ $\left(4 \mathrm{CH}, \mathrm{C} 2\right.$ ' and C6'), $122.9\left(2 \mathrm{CH}, \mathrm{C}^{\prime}\right), 128.6(\mathrm{CH}), 129.1(4 \mathrm{CH}, \mathrm{C} 3$ ' and C5'), 130.3 (CH), 147.7 (2C, C1'), 149.8 (C, C3), 161.3 (C, C=O). HRMS (EI) $\mathrm{m} / \mathrm{z}$ calcd for $\mathrm{C}_{18} \mathrm{H}_{15} \mathrm{NO}_{2} \mathrm{~S}: 309.0823$; found: 309.0820 .

\section{Methyl 3-(di(3-thienyl)amino)-2-thiophenecarboxylate (10d).}

The general procedure 7 using 3-iodothiophene $(0.20 \mathrm{~mL})$ gave $10 \mathbf{d}$ (eluent: hexanes-AcOEt $\left.90: 10 ; R_{f}=0.52\right)$ in $87 \%$ yield $(0.28 \mathrm{~g})$ as a yellow oil; IR (ATR): 605, 626, 649, 726, 749, 761, 841, 914, 986, 1042, $1073,1099,1151,1188,1214,1297,1375,1401,1423,1437,1520$, 1692, 1713, 2947, $3105 \mathrm{~cm}^{-1} ;{ }^{1} \mathrm{H}$ NMR $\left(300 \mathrm{MHz}, \mathrm{CDCl}_{3}\right) \delta 3.59(\mathrm{~s}, 3 \mathrm{H}$ $\mathrm{OMe}$ ), 6.58 (dd, $2 \mathrm{H}, J=3.1$ and $1.2 \mathrm{~Hz}, \mathrm{H} 2$ '), $6.86(\mathrm{dd}, 2 \mathrm{H}, J=5.2$ and $1.2 \mathrm{~Hz}, \mathrm{H} 4^{\prime}$ ), 6.88 (d, $\left.1 \mathrm{H}, J=5.6 \mathrm{~Hz}, \mathrm{H} 4\right), 7.18$ (dd, $2 \mathrm{H}, J=5.2,3.2 \mathrm{~Hz}$, $\mathrm{H} 5$ '), $7.40(\mathrm{~d}, 1 \mathrm{H}, \mathrm{J}=5.3 \mathrm{~Hz}, \mathrm{H} 5) ;{ }^{13} \mathrm{C} \mathrm{NMR}\left(75 \mathrm{MHz} \mathrm{CDCl}_{3}\right) \delta 51.8$ $\left(\mathrm{CH}_{3}\right), 109.7(2 \mathrm{CH}), 120.0(\mathrm{C}), 123.3(2 \mathrm{CH}), 124.8(2 \mathrm{CH}), 127.3(\mathrm{CH})$ $130.2(\mathrm{CH}), 146.8$ (2C, C3'), 149.9 (C), 161.4 (C, C=O). HRMS (El) $\mathrm{m} / \mathrm{z}$ calcd for $\mathrm{C}_{14} \mathrm{H}_{11} \mathrm{NO}_{2} \mathrm{~S}_{3}$ : 320.9952; found: 320.9957. Methyl 3-(3thienylamino)-2-thiophenecarboxylate (9d) was similarly isolated (eluent: hexanes-AcOEt $\left.90: 10 ; R_{f}=0.575\right)$ in $<10 \%$ yield as a yellow oil: IR (ATR): 455, 584, 610, 722, 770, 840, 958, 1035, 1083, 1238, 1350, 1402, 1424, 1444, 1556, 1571, 1661, 2949, 3103, $3330 \mathrm{~cm}^{-1} ;{ }^{1} \mathrm{H}$ NMR $\left(300 \mathrm{MHz} \mathrm{CDCl}_{3}\right) \delta 3.86(\mathrm{~s}, 3 \mathrm{H}, \mathrm{OMe}), 6.82(\mathrm{dd}, 1 \mathrm{H}, J=3.2,1.4 \mathrm{~Hz}, \mathrm{H} 2$ '), 6.95 (dd, $1 \mathrm{H}, J=5.2,1.4 \mathrm{~Hz}, \mathrm{H} 4$ ') 7.03 (d, 1H, J = 5.5 Hz, H4), 7.27 (dd, $1 \mathrm{H}, J=5.1,3.1 \mathrm{~Hz}, \mathrm{H} 5$ ) $, 7.36(\mathrm{~d}, 1 \mathrm{H}, J=5.5 \mathrm{~Hz}, \mathrm{H} 5), 8.75$ (br s, $1 \mathrm{H}$, $\mathrm{NH}) ;{ }^{13} \mathrm{C}$ NMR $\left(75 \mathrm{MHz}, \mathrm{CDCl}_{3}\right) \delta 51.5\left(\mathrm{CH}_{3}\right), 102.0(\mathrm{C}), 109.1(\mathrm{CH})$, $118.0(\mathrm{CH}), 123.4(\mathrm{CH}), 125.5(\mathrm{CH}), 132.1(\mathrm{CH}), 140.4(\mathrm{C}), 152.4(\mathrm{C})$, $165.3(\mathrm{C})$.

General procedure 8 for the $\mathrm{N}$-arylation of methyl 3-(phenylamino)2-thiophenecarboxylate (9a).

To the required iodide $(1.0 \mathrm{mmol})$ in $\mathrm{Bu}_{2} \mathrm{O}(1.5 \mathrm{~mL})$ were successively added activated $\mathrm{Cu}$ (13 $\mathrm{mg}, 0.20 \mathrm{mmol})$, methyl 3-(phenylamino)-2thiophenecarboxylate $(9 \mathrm{a}, 0.23 \mathrm{~g}, 1.0 \mathrm{mmol})$ and $\mathrm{K}_{2} \mathrm{CO}_{3}(0.28 \mathrm{~g}$, $2.0 \mathrm{mmol}$ ). The mixture was degassed and heated at $1400^{\circ} \mathrm{C}$ under argon for $24 \mathrm{~h}$. During this time, activated $\mathrm{Cu}(4 \times 13 \mathrm{mg}, 4 \times 0.20 \mathrm{mmol})$ was 
added after 2, 4, 6 and $8 \mathrm{~h}$ of heating. After cooling to room temperature, the mixture was concentrated. Addition of $\mathrm{H}_{2} \mathrm{O}(25 \mathrm{~mL})$, extraction with AcOEt $(3 \times 10 \mathrm{~mL})$, drying over $\mathrm{Na}_{2} \mathrm{SO}_{4}$, removal of the solvent and purification by chromatography on silica gel (the eluent is given in the product description) led to the expected compound.

\section{Methyl}

$\mathrm{N}$-(4-methoxyphenyl)- $\mathrm{N}$-phenyl-3-amino-2 thiophenecarboxylate (10ab). The general procedure 8 using 4 iodoanisole $(0.23 \mathrm{~g})$ gave 10ab (eluent: hexanes-AcOEt 95:5; $R_{f}=0.27$ ) in $91 \%$ yield $(0.31 \mathrm{~g})$ as a yellow powder: $\mathrm{mp} 122{ }^{\circ} \mathrm{C}$; IR (ATR): 665,693 $720,747,769,779,789,805,836,1026,1041,1073,1098,1225,1247$, 1258, 1442, 1489, 1505, 1594, 1709, 2922, $2957 \mathrm{~cm}^{-1} ;{ }^{1} \mathrm{H}$ NMR (300 $\left.\mathrm{MHz} \mathrm{CDCl}_{3}\right) \delta 3.53$ (s, 3H, OMe), 3.79 (s, 3H, OMe), 6.77 (d, $1 \mathrm{H}, J=5.4$ $\mathrm{Hz}, \mathrm{H} 4), 6.81$ (d, 2H, J = 9.0 Hz), 6.90-6.95 (m, 3H, H2', H4' and H6'), $7.03(\mathrm{~d}, 2 \mathrm{H}, J=8.9 \mathrm{~Hz}), 7.19\left(\mathrm{t}, 2 \mathrm{H}, J=7.9 \mathrm{~Hz}, \mathrm{H} 3^{\prime}\right.$ and H5'), $7.39(\mathrm{~d}, 1 \mathrm{H}$ $J=5.4 \mathrm{~Hz}, \mathrm{H} 5) ;{ }^{13} \mathrm{C}$ NMR $\left(75 \mathrm{MHz}, \mathrm{CDCl}_{3}\right) \delta 51.7\left(\mathrm{CH}_{3}\right), 55.6\left(\mathrm{CH}_{3}\right)$, $114.6(2 \mathrm{CH}), 119.9(\mathrm{C}), 121.1(2 \mathrm{CH}), 122.0(\mathrm{CH}), 125.8(2 \mathrm{CH}), 128.3$ $(\mathrm{CH}), 129.0(2 \mathrm{CH}), 130.2(\mathrm{CH}), 140.8(\mathrm{C}), 148.6(\mathrm{C}), 150.2(\mathrm{C}), 156.3(\mathrm{C})$ $161.3(\mathrm{C})$; HRMS (EI) $\mathrm{m} / z$ calcd for $\mathrm{C}_{19} \mathrm{H}_{17} \mathrm{NO}_{3} \mathrm{~S}$ : 339.0929 ; found: 339.0926. Crystal data for $10 \mathrm{ab} . \mathrm{C}_{19} \mathrm{H}_{17} \mathrm{NO}_{3} \mathrm{~S}, \mathrm{M}=339.39$, triclinic, $P-1$, $a=9.2351(10), b=9.7922(11), c=9.9542(11) \AA, \alpha=102.222(4), \beta=$ 96.641(4), $\gamma=108.875(4)^{\circ}, V=815.80(16) \AA^{3}, Z=2, d=1.382 \mathrm{~g} \mathrm{~cm}^{-3}, \mu$ $=0.215 \mathrm{~mm}^{-1}$. A final refinement on $F^{2}$ with 3725 unique intensities and 219 parameters converged at $\omega R\left(F^{2}\right)=0.0824(R(F)=0.0333)$ for 3289 observed reflections with $I>2 \sigma(I)$. CCDC 1896368

Methyl N-phenyl-N-(4-(trifluoromethyl)phenyl)-3-amino-2thiophenecarboxylate (10ac). The general procedure 8 using 1-iodo-4 (trifluoromethyl)benzene $(0.15 \mathrm{~mL})$ gave 10ac (eluent: hexanes-AcOEt $\left.95: 5 ; R_{f}=0.40\right)$ in $94 \%$ yield $(0.35 \mathrm{~g})$ as a yellow oil: IR $(A T R): 508,522$, $611,646,684,698,732,749,769,838,951,1011,1040,1066,1110$, $1161,1183,1226,1279,1297,1316,1396,1438,1490,1515,1528$, 1591, 1614, 1715, $2951 \mathrm{~cm}^{-1} ;{ }^{1} \mathrm{H}$ NMR $\left(300 \mathrm{MHz}, \mathrm{CDCl}_{3}\right) \delta 3.59(\mathrm{~s}, 3 \mathrm{H}$, OMe), $6.87(\mathrm{~d}, 1 \mathrm{H}, J=5.3 \mathrm{~Hz}, \mathrm{H} 4), 7.03(\mathrm{~d}, 2 \mathrm{H}, J=8.5 \mathrm{~Hz}), 7.09-7.15(\mathrm{~m}$ $3 \mathrm{H}), 7.31$ (t, $2 \mathrm{H}, J=7.8 \mathrm{~Hz}, \mathrm{H} 3^{\prime}$ and $\left.\mathrm{H} 5^{\prime}\right), 7.45(\mathrm{~d}, 2 \mathrm{H}, J=8.6 \mathrm{~Hz}), 7.50$ $(\mathrm{d}, 1 \mathrm{H}, J=5.3 \mathrm{~Hz}, \mathrm{H} 5) ;{ }^{13} \mathrm{C}$ NMR $\left(75 \mathrm{MHz}, \mathrm{CDCl}_{3}\right) \delta 51.8\left(\mathrm{CH}_{3}\right), 119.9$ $(2 \mathrm{CH}), 123.1(\mathrm{C}), 123.2$ (q, C, J = 32.4 Hz, C4"), $124.2(2 \mathrm{CH}), 124.5(\mathrm{CH})$ 124.6 (q, C, $\left.J=269 \mathrm{~Hz}, \mathrm{CF}_{3}\right), 126.2$ (q, 2CH, J=3.7 Hz, C3" and C5"), $128.7(\mathrm{CH}), 129.4(2 \mathrm{CH}), 130.9(\mathrm{CH}), 146.2(\mathrm{C}), 148.5(\mathrm{C}), 150.8(\mathrm{~d}, \mathrm{C}, \mathrm{J}$ $=1.3 \mathrm{~Hz}), 160.9(\mathrm{C})$. HRMS (EI) $\mathrm{m} / z$ calcd for $\mathrm{C}_{19} \mathrm{H}_{14} \mathrm{~F}_{3} \mathrm{NO}_{2} \mathrm{~S}: 377.0697$; found: 377.0698 .

Methyl 2-(phenylamino)-3-thiophenecarboxylate (11a). To iodobenzene $(0.11 \mathrm{~mL}, 1.0 \mathrm{mmol})$ in $\mathrm{Bu}_{2} \mathrm{O}(1.5 \mathrm{~mL})$ were successively added activated $\mathrm{Cu}(13 \mathrm{mg}, 0.20 \mathrm{mmol})$, methyl 2-amino-3thiophenecarboxylate $(0.16 \mathrm{~g}, 1.0 \mathrm{mmol})$ and $\mathrm{K}_{2} \mathrm{CO}_{3}(0.28 \mathrm{~g}, 2.0 \mathrm{mmol})$. The mixture was degassed and heated at $140{ }^{\circ} \mathrm{C}$ under argon for $24 \mathrm{~h}$. During this time, activated $\mathrm{Cu}(4 \times 13 \mathrm{mg}, 4 \times 0.20 \mathrm{mmol})$ was added after 2, 4, 6 and $8 \mathrm{~h}$ of heating. After cooling to room temperature, the mixture was concentrated. Addition of $\mathrm{H}_{2} \mathrm{O}(25 \mathrm{~mL})$, extraction with AcOEt $(3 \times 10 \mathrm{~mL})$, drying over $\mathrm{Na}_{2} \mathrm{SO}_{4}$, removal of the solvent and purification by chromatography on silica gel (eluent: hexanes-AcOEt 95:5; $\left.R_{\mathrm{f}}=0.50\right)$ afforded $11 \mathrm{a}$ in $83 \%$ yield $(0.19 \mathrm{~g})$ as a white powder: $\mathrm{mp}$ $64^{\circ} \mathrm{C}$ (lit. ${ }^{[3]} 59-60{ }^{\circ} \mathrm{C}$ ); IR (ATR): 490, 503, 661, 684, 751, 782, 1012, 1084, 1154, 1193, 1241, 1337, 1382, 1407, 1440, 1498, 1509, 1553 , 1592, 1670, 2948, $3213 \mathrm{~cm}^{-1}$; ${ }^{1} \mathrm{H}$ NMR $\left(300 \mathrm{MHz}, \mathrm{CDCl}_{3}\right) \delta 3.87$ (s, 3H, OMe), $6.31(\mathrm{~d}, 1 \mathrm{H}, J=5.7 \mathrm{~Hz}), 7.07\left(\mathrm{t}, 1 \mathrm{H}, J=7.1 \mathrm{~Hz}, \mathrm{H} 4^{\prime}\right), 7.15$ (d, 1H, $J=5.8 \mathrm{~Hz}$ ), 7.31 (d, 2H, $J=7.6 \mathrm{~Hz}, \mathrm{H} 2^{\prime}$ and H6'), $7.37(\mathrm{t}, 2 \mathrm{H}, J=7.8 \mathrm{~Hz}$ H3' and H5'), 9.91 (br s, $1 \mathrm{H}, \mathrm{NH}$ ); ${ }^{13} \mathrm{C} \mathrm{NMR}\left(75 \mathrm{MHz} \mathrm{CDCl}_{3}\right) \delta 51.3$ $\left(\mathrm{CH}_{3}\right), 107.1(\mathrm{CH}), 107.5(\mathrm{C}), 118.2(2 \mathrm{CH}), 122.9(\mathrm{CH}), 125.4(\mathrm{CH})$, $129.5(2 \mathrm{CH}), 140.9(\mathrm{C}), 158.5(\mathrm{C}), 166.4(\mathrm{C})$. These data are similar to those described previously. ${ }^{[37]}$
General procedure 9 for the bis- $\mathrm{N}$-arylation of methyl 2-amino-3thiophenecarboxylate using the same iodide.

To the required iodide $(2.0 \mathrm{mmol})$ in $\mathrm{Bu}_{2} \mathrm{O}(1.5 \mathrm{~mL})$ were successively added activated $\mathrm{Cu}(13 \mathrm{mg}, 0.20 \mathrm{mmol})$, methyl 2-amino-3thiophenecarboxylate $(0.16 \mathrm{~g}, 1.0 \mathrm{mmol})$ and $\mathrm{K}_{2} \mathrm{CO}_{3}(0.28 \mathrm{~g}, 2.0 \mathrm{mmol})$. The mixture was degassed and heated at $140{ }^{\circ} \mathrm{C}$ under argon for $24 \mathrm{~h}$. During this time, activated $\mathrm{Cu}(4 \times 13 \mathrm{mg}, 4 \times 0.20 \mathrm{mmol})$ was added after 2, 4, 6 and $8 \mathrm{~h}$ of heating. After cooling to room temperature, the mixture was concentrated. Addition of $\mathrm{H}_{2} \mathrm{O}(25 \mathrm{~mL})$, extraction with AcOEt $(3 \times 10 \mathrm{~mL})$, drying over $\mathrm{Na}_{2} \mathrm{SO}_{4}$, removal of the solvent and purification by chromatography on silica gel (the eluent is given in the product description) led to the expected compound.

\section{Methyl 2-(diphenylamino)-3-thiophenecarboxylate (12a).}

The general procedure 9 using iodobenzene $(0.22 \mathrm{~mL})$ gave $12 \mathrm{a}$ (eluent: hexanes-AcOEt $\left.95: 5 ; R_{f}=0.27\right)$ in $54 \%$ yield $(0.17 \mathrm{~g})$ as a yellow powder: mp $110{ }^{\circ} \mathrm{C} ;{ }^{1} \mathrm{H}$ NMR $\left(300 \mathrm{MHz}, \mathrm{CDCl}_{3}\right) \delta 3.52$ (s, 3H, OMe), $6.98\left(\mathrm{~d}, 1 \mathrm{H}, J=5.9 \mathrm{~Hz}\right.$ ), 7.03 (t, $\left.2 \mathrm{H}, J=7.5 \mathrm{~Hz}, \mathrm{H} 4^{\prime}\right), 7.08$ (d, 4H, J= 7.9 Hz, H2' and H6'), 7.25 (t, 4H, J=7.8 Hz, H3' and H5'), 7.33 (d, 1H, J= $5.9 \mathrm{~Hz}) ;{ }^{13} \mathrm{C}$ NMR $\left(75 \mathrm{MHz}, \mathrm{CDCl}_{3}\right) \delta 51.4\left(\mathrm{CH}_{3}\right), 120.1(\mathrm{CH}), 122.5$ $(4 \mathrm{CH}), 123.2(2 \mathrm{CH}), 124.7(\mathrm{C}), 128.2(\mathrm{CH}), 129.1(4 \mathrm{CH}), 147.9(2 \mathrm{C})$, 156.8 (C), 162.5 (C). HRMS (EI) $\mathrm{m} / \mathrm{z}$ calcd for $\mathrm{C}_{18} \mathrm{H}_{15} \mathrm{NO}_{2} \mathrm{~S}: 309.0823$ found: 309.0819

\section{Methyl 2-(di(3-thienyl)amino)-3-thiophenecarboxylate (12d).}

The general procedure 9 using 3-iodothiophene $(0.20 \mathrm{~mL})$ gave $12 \mathbf{d}$ (eluent: hexanes-AcOEt $\left.90: 10 ; R_{f}=0.52\right)$ in $76 \%$ yield $(0.24 \mathrm{~g})$ as a yellow oil: IR (ATR): 472, 601, 630, 637, 668, 694, 734, 761, 782, 829, $836,846,871,970,997,1072,1083,1129,1148,1185,1237,1256$ $1285,1383,1395,1453,1522,1537,1695,2949,3104 \mathrm{~cm}^{-1} ;{ }^{1} \mathrm{H}$ NMR $\left(300 \mathrm{MHz} \mathrm{CDCl}_{3}\right) \delta 3.59(\mathrm{~s}, 3 \mathrm{H}, \mathrm{OMe}), 6.64(\mathrm{~d}, 2 \mathrm{H}, J=2.4 \mathrm{~Hz}, \mathrm{H} 2$ '), 6.89 (d, 2H, J = 5.2 Hz, H4'), 6.93 (d, 1H, J = 5.9 Hz), 7.18 (dd, 2H, J = 5.1 and $3.2 \mathrm{~Hz}, \mathrm{H} 5$ '), $7.32(\mathrm{~d}, 1 \mathrm{H}, J=5.9 \mathrm{~Hz}) ;{ }^{13} \mathrm{C} \mathrm{NMR}\left(75 \mathrm{MHz} \mathrm{CDCl}_{3}\right) \delta$ $51.3\left(\mathrm{CH}_{3}\right), 109.9(2 \mathrm{CH}), 119.4(\mathrm{CH}), 122.7(2 \mathrm{CH}), 123.6(\mathrm{C}), 124.8$ $(2 \mathrm{CH}), 127.9(\mathrm{CH}), 146.7(\mathrm{C}), 156.5(\mathrm{C}), 162.2(\mathrm{C})$. HRMS (EI) $\mathrm{m} / \mathrm{z}$ calcd for $\mathrm{C}_{14} \mathrm{H}_{11} \mathrm{NO}_{2} \mathrm{~S}_{3}$ : 320.9952; found: 320.9948 .

General procedure 10 for the $\mathbf{N}$-arylation of methyl 2-(phenylamino)3-thiophenecarboxylate (11a)

To the required iodide $(1.0 \mathrm{mmol})$ in $\mathrm{Bu}_{2} \mathrm{O}(1.5 \mathrm{~mL})$ were successively added activated $\mathrm{Cu}(13 \mathrm{mg}, 0.20 \mathrm{mmol})$, methyl 2-(phenylamino)-3thiophenecarboxylate $(11 \mathrm{a}, 0.23 \mathrm{~g}, 1.0 \mathrm{mmol})$ and $\mathrm{K}_{2} \mathrm{CO}_{3}(0.28 \mathrm{~g}$, $2.0 \mathrm{mmol}$ ). The mixture was degassed and heated at $140{ }^{\circ} \mathrm{C}$ under argon for $24 \mathrm{~h}$. During this time, activated $\mathrm{Cu}(4 \times 13 \mathrm{mg}, 4 \times 0.20 \mathrm{mmol})$ was added after 2, 4, 6 and $8 \mathrm{~h}$ of heating. After cooling to room temperature, the mixture was concentrated. Addition of $\mathrm{H}_{2} \mathrm{O}(25 \mathrm{~mL})$, extraction with AcOEt $(3 \times 10 \mathrm{~mL})$, drying over $\mathrm{Na}_{2} \mathrm{SO}_{4}$, removal of the solvent and purification by chromatography on silica gel (the eluent is given in the product description) led to the expected compound.

\section{Methyl thiophenecarboxylate (12ab) \\ $\mathrm{N}$-(4-methoxyphenyl)- $\mathrm{N}$-phenyl-2-amino-3-}

The general procedure 10 using 4-iodoanisole $(0.23 \mathrm{~g})$ gave 12ab (eluent: hexanes-AcOEt $\left.90: 10 ; R_{f}=0.25\right)$ in $86 \%$ yield $(0.29 \mathrm{~g}$ ) as a red oil: IR (ATR): 533, 570, 614, 632, 645, 694, 750, 823, 910, 1003, 1033 , 1084, 1108, 1144, 1191, 1238, 1284, 1387, 1438, 1463, 1490, 1506, 1531, 1594, 1704, 2835, $2949 \mathrm{~cm}^{-1} ;{ }^{1} \mathrm{H}$ NMR (300 MHz, $\left.\mathrm{CDCl}_{3}\right) \delta 3.55$ (s, $3 \mathrm{H}, \mathrm{OMe}$ ), 3.79 (s, 3H, OMe), 6.86 (d, 2H, J = 8.9 Hz, H2" and H6"), 6.91 
$(\mathrm{d}, 1 \mathrm{H}, J=5.9 \mathrm{~Hz}), 6.95-6.99(\mathrm{~m}, 3 \mathrm{H}), 7.15(\mathrm{~d}, 2 \mathrm{H}, J=8.9 \mathrm{~Hz}, \mathrm{H3}$ " and $\mathrm{H} 5$ "), 7.18-7.24 (m, 2H), $7.32(\mathrm{~d}, 1 \mathrm{H}, J=5.9 \mathrm{~Hz}) ;{ }^{13} \mathrm{C} \mathrm{NMR}(75 \mathrm{MHz}$, $\left.\mathrm{CDCl}_{3}\right) \delta 51.2\left(\mathrm{CH}_{3}\right), 55.4\left(\mathrm{CH}_{3}\right), 114.5(2 \mathrm{CH}), 119.3(\mathrm{CH}), 120.3(2 \mathrm{CH})$, $121.9(\mathrm{CH}), 123.4(\mathrm{C}), 125.8(2 \mathrm{CH}), 128.0(\mathrm{CH}), 128.9(2 \mathrm{CH}), 140.7(\mathrm{C})$, 148.6 (C), 156.6 (C), 157.5 (C), 162.3 (C) ; HRMS (EI) $\mathrm{m} / \mathrm{z}$ calcd for $\mathrm{C}_{19} \mathrm{H}_{17} \mathrm{NO}_{3} \mathrm{~S}$ : 339.0929 ; found: 339.0932 .

\section{Methyl $\quad \mathrm{N}$-(2-benzofuryl)- $\mathrm{N}$-phenyl-2-amino-3-thiophenecarboxylate (12ae).}

The general procedure 10 using 2-iodobenzofuran $(0.24 \mathrm{~g})$ gave 12ae (eluent: hexanes-AcOEt $\left.90: 10 ; R_{f}=0.425\right)$ in $53 \%$ yield $(0.18 \mathrm{~g})$ as an orange oil: IR (ATR): 486, 501, 692, 708, 744, 764, 828, 908, 971, 1006, $1084,1105,1153,1152,1211,1248,1281,1342,1389,1437,1452$, $1492,1536,1582,1709,2949,3062 \mathrm{~cm}^{-1} ;{ }^{1} \mathrm{H}$ NMR $\left(300 \mathrm{MHz}, \mathrm{CDCl}_{3}\right) \delta$ 3.61 (s, 3H, OMe), $6.11(\mathrm{~s}, 1 \mathrm{H}, \mathrm{H} 3$ "), $7.09(\mathrm{~d}, 1 \mathrm{H}, J=5.9 \mathrm{~Hz}), 7.11-7.22$ $(\mathrm{m}, 5 \mathrm{H}), 7.31-7.39(\mathrm{~m}, 3 \mathrm{H}), 7.42-7.45(\mathrm{~m}, 2 \mathrm{H}) ;{ }^{13} \mathrm{C} \mathrm{NMR}\left(75 \mathrm{MHz}, \mathrm{CDCl}_{3}\right)$

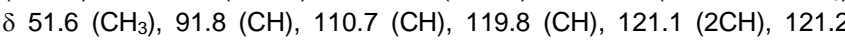
$(\mathrm{CH}), 122.8(\mathrm{CH}), 123.1(\mathrm{CH}), 124.0(\mathrm{CH}), 125.9(\mathrm{C}), 128.2(\mathrm{CH}), 129.2$ (2CH), $129.3(\mathrm{C}), 145.6(\mathrm{C}), 151.3(\mathrm{C}), 152.5(\mathrm{C}), 155.2(\mathrm{C}), 162.2(\mathrm{C})$. HRMS (EI) $\mathrm{m} / z$ calcd for $\mathrm{C}_{20} \mathrm{H}_{15} \mathrm{NO}_{3} \mathrm{~S}$ : 349.0773 ; found: 349.0770 .

\section{Acknowledgements}

We thank the Université de Rennes 1, and the Centre National de la Recherche Scientifique (F. M.). We acknowledge the Fonds Européen de Développement Régional (FEDER; D8 VENTURE Bruker AXS diffractometer) and Thermofisher (generous gift of 2,2,6,6-tetramethylpiperidine). This research has been partly performed as part of the CNRS PICS project "Bimetallic synergy for the functionalization of heteroaromatics". Maria Ivanova thanks the Alexander von Humboldt foundation for a fellowship.

Keywords: thiophene $\cdot$ copper $\cdot$ cobalt $\cdot$ zinc $\cdot \mathrm{C}-\mathrm{N}$ bond formation

[1] E. R. Biehl, Top. Heterocycl. Chem. 2012, 29, 347-380.

[2] D. C. Blakemore, L. Castro, I. Churcher, D. C. Rees, A. W. Thomas, D. M. Wilson, A. Wood, Nat. Chem. 2018, 10, 383-394.

[3] a) J. F. Hartwig, Acc. Chem. Res. 2008, 41, 1534-1544; b) J. F. Hartwig, Nature 2008, 455, 314-322; c) Y. Aubin, C. Fischmeister, C. M. Thomas J.-L. Renaud, Chem. Soc. Rev. 2010, 39, 4130-4145; d) I. P. Beletskaya, A. V. Cheprakov, Organometallics 2012, 31, 7753-7808; e) J. Bariwal, E. Van der Eycken, Chem. Soc. Rev. 2013, 42, 9283-9303; f) J. Kim, H. J. Kim, S. Chang, Eur. J. Org. Chem. 2013, 2013, 32013213; g) J. Schranck, A. Tlili, ACS Catal. 2018, 8, 405-418.

[4] a) D. S. Surry, S. L. Buchwald, Chem. Sci. 2011, 2, 27-50; b) R. J. Lundgren, M. Stradiotto, Aldrichimica Acta 2012, 45, 59-65; c) P. RuizCastillo, S. L. Buchwald, Chem. Rev. 2016, 116, 12564-12649.

[5] a) F. Monnier, M. Taillefer, Angew. Chem. 2008, 120, 3140-3143; Angew. Chem. Int. Ed. 2009, 48, 6954-6971; b) F. Monnier, M. Taillefer, Top. Organomet. Chem. 2013, 46, 173-204; c) P. J. Amal Joseph, S. Priyadarshini, Org. Process Res. Dev. 2017, 21, 1889-1924.

[6] a) M. Zhang, Synthesis 2011, 3408-3417; b) C. E. Hendrick, Q. Wang, J. Org. Chem. 2017, 82, 839-847.

[7] a) T. W. Lyons, M. S. Sanford, Chem. Rev. 2010, 110, 1147-1169; b) J. Yamaguchi, A. D. Yamaguchi, K. Itami, Angew. Chem. 2012, 124, 9092-9142; Angew. Chem. Int. Ed. 2012, 51, 8960-9009; c) M.-L. Louillat, F. W. Patureau, Chem. Soc. Rev. 2014, 43, 901-910; d) H. Kim,
S. Chang, ACS Catal. 2016, 6, 2341-2351; e) J. Jiao, K. Murakami, K Itami, ACS Catal. 2016, 6, 610-633; f) Y. Park, Y. Kim, S. Chang, Chem. Rev. 2017, 117, 9247-9301.

[8] a) X. Yan, X. Yang, C. Xi, Catal. Sci. Technol. 2014, 4, 4169-4177; b) M. Corpet, C. Gosmini, Synthesis 2014, 46, 2258-2271; c) X. Dong, Q. Liu Y. Dong, H. Liu, Chem. Eur. J. 2017, 23, 2481-2511.

[9] L. Bianchi, M. Maccagno, G. Petrillo, F. Sancassan, C. Tavani, S. Morganti, E. Rizzato, D. Spinelli, J. Org. Chem. 2007, 72, 5771-5777.

[10] a) M. Kienle, A. J. Wagner, C. Dunst, P. Knochel, Chem. Asian J. 2011, 6, 517-523; b) C. Dunst, M. Kienle, P. Knochel, Synthesis 2010, 2313 2318.

[11] A. M. Martínez, N. Rodríguez, R. G. Arrayás, J. C. Carretero, Chem Commun. 2014, 50, 2801-2803.

[12] B. Berzina, I. Sokolovs, E. Suna, ACS Catal. 2015, 5, 7008-7014

[13] M. Shang, S.-H. Zeng, S.-Z. Sun, H.-X. Dai, J.-Q. Yu, Org. Lett. 2013, 15, 5286-5289.

[14] H. Yoon, Y. Lee, J. Org. Chem. 2015, 80, 10244-10251.

[15] a) S. L. McDonald, C. E. Hendrick, Q. Wang, Angew. Chem. 2014, 126 4755-4758; Angew. Chem. Int. Ed. 2014, 53, 4667-4670; b) S. L. McDonald, C. E. Hendrick, K. J. Bitting, Q. Wang, Organic Syntheses 2015, 92, 356-372; c) C. E. Hendrick, K. J. Bitting, S. Cho, Q. Wang, J. Am. Chem. Soc. 2017, 139, 11622-11628.

[16] A. M. Berman, J. S. Johnson, J. Am. Chem. Soc. 2004, 126, 5680-5681. We used the protocol reported inside to prepare our starting O-benzoyl hydroxylamines.

[17] Y.-H. Chen, S. GraßI, P. Knochel, Angew. Chem. 2018, 130, 1120 1124; Angew. Chem. Int. Ed. 2018, 57, 1108-1111. See Supporting Information for a suggestion concerning the role of TMEDA in the reactions displayed in Scheme 4.

[18] S. Graßı, Y.-H. Chen, C. Hamze, C. P. Tüllmann, P. Knochel, Org. Lett. 2019, 21, 494-497

[19] For the synthesis of $\mathrm{ZnCl}_{2} \cdot$ TMEDA, see: R. A. Kjonaas, R. K. Hoffer, J. Org. Chem. 1988, 53, 4133-4135. See also: K. Snégaroff, S. Komagawa, F. Chevallier, P. C. Gros, S. Golhen, T. Roisnel, M. Uchiyama, F. Mongin, Chem. Eur. J. 2010, 16, 8191-8201.

[20] N. Mokhtari Brikci-Nigassa, G. Bentabed-Ababsa, W. Erb, F. Mongin Synthesis 2018, 50, 3615-3633.

[21] See for example: a) Z. Lu, R. J. Twieg, Tetrahedron 2005, 61, 903-918; b) S. Fantasia, J. Windisch, M. Scalone, Adv. Synth. Catal. 2013, 355, 627-631; c) F. Dierschke, J. Jacob, A. K. Mishra, A. C. Grimsdale, K. Mullen, Polym. Prepr. 2004, 45, 170-171.

[22] M. Balkenhohl, R. Greiner, I. S. Makarov, B. Heinz, K. Karaghiosoff, H. Zipse, P. Knochel, Chem. Eur. J. 2017, 23, 13046-13050, and references cited therein.

[23] Y. Hamada, M. Sato, I. Takeuchi, Yakugaku Zasshi 1975, 95, $1492-$ 1497

[24] See for example: E. A. Voight, H. Yin, S. V. Downing, S. A. Calad, H Matsuhashi, I. Giordano, A. J. Hennessy, R. M. Goodman, J. L. Wood, Org. Lett. 2010, 12, 3422-3425.

[25] M. Mosrin, P. Knochel, Org. Lett. 2009, 11, 1837-1840.

[26] A. Krasovskiy, V. Krasovskaya, P. Knochel, Angew. Chem. 2006, 118 3024-3027; Angew. Chem. Int. Ed. 2006, 45, 2958-2961.

[27] Concerning amination of organomagnesium compounds, see: M. J. Campbell, J. S. Johnson, Org. Lett. 2007, 9, 1521-1524.

[28] S. M. Manolikakes, M. Ellwart, C. I. Stathakis, P. Knochel, Chem. Eur. J. 2014, 20, 12289-12297.

[29] A. Krasovskiy, P. Knochel, Angew. Chem. 2004, 116, 3396-3399; Angew. Chem. Int. Ed. 2004, 43, 3333-3336.

[30] See for example: J. M. Hammann, D. Haas, P. Knochel, Angew. Chem 2015, 127, 4560-4563; Angew. Chem. Int. Ed. 2015, 54, 4478-4481.

[31] T. Kunz, P. Knochel, Chem. Eur. J. 2011, 17, 866-872.

[32] J. Nafe, P. Knochel, Synthesis 2016, 48, 103-114

[33] a) K. R. Hornberger, J. G. Badiang, J. M. Salovich, K. W. Kuntz, K. A Emmitte, M. Cheung, Tetrahedron Lett. 2008, 49, 6348-6351; b) V. E. Laing, D. C. Brookings, R. J. Carbery, J. G. Simorte, M. C. Hutchings, B. 
J. Langham, M. A. Lowe, R. A. Allen, J. R. Fetterman, J. Turner, C. Meier, J. Kennedy, M. Merriman, Bioorg. Med. Chem. Lett. 2012, 22 472-475.

[34] a) J. Yin, M. M. Zhao, M. A. Huffman, J. M. McNamara, Org. Lett. 2002 4, 3481-3484; b) J. Hartwig, S. Ceylan, L. Kupracz, L. Coutable, A Kirschning, Angew. Chem. 2013, 125, 9995-9999; Angew. Chem. Int Ed. 2013, 52, 9813-9817; c) N. Saadatjoo, M. Javaheri, N. Saemian, M. Amini, J. Labelled Compd. Radiopharm. 2016, 59, 325-327; d) D. Obermayer, D. Znidar, G. Glotz, A. Stadler, D. Dallinger, C. O. Kappe, J. Org. Chem. 2016, 81, 11788-11801.

[35] a) A. Correa, I. Tellitu, E. Dominguez, R. SanMartin, Tetrahedron 2006, 62, 11100-11105; b) M.-J. R. P. Queiroz, R. C. Calhelha, G. Kirsch, Tetrahedron 2007, 63, 13000-13005; c) M. Carril, R. SanMartin, E. Dominguez, I. Tellitu, Tetrahedron 2007, 63, 690-702; d) R. C. Calhelha M.-J. R. P. Queiroz, Tetrahedron Lett. 2010, 51, 281-283; e) A. J. Buckmelter, L. Ren, E. R. Laird, B. Rast, G. Miknis, S. Wenglowsky, S. Schlachter, M. Welch, E. Tarlton, J. Grina, J. Lyssikatos, B. J. Brandhuber, T. Morales, N. Randolph, G. Vigers, M. Martinson, M. Callejo, Bioorg. Med. Chem. Lett. 2011, 21, 1248-1252; f) R. C. Calhelha, I. C. F. R. Ferreira, D. Peixoto, R. M. V. Abreu, L. A. ValeSilva, E. Pinto, R. T. Lima, M. I. Alvelos, M. H. Vasconcelos, M.-J. R. P. Queiroz, Molecules 2012, 17, 3834-3843.

[36] a) M. Hedidi, W. Erb, G. Bentabed-Ababsa, F. Chevallier, L. Picot, V. Thiery, S. Bach, S. Ruchaud, T. Roisnel, V. Dorcet, F. Mongin, Tetrahedron 2016, 72, 6467-6476; b) M. Hedidi, J. Maillard, W. Erb, F. Lassagne, Y. S. Halauko, O. A. Ivashkevich, V. E. Matulis, T. Roisnel, V. Dorcet, M. Hamze, Z. Fajloun, B. Baratte, S. Ruchaud, S. Bach, G. Bentabed-Ababsa, F. Mongin, Eur. J. Org. Chem. 2017, 5903-5915; c) R. Amara, G. Bentabed-Ababsa, M. Hedidi, J. Khoury, H. Awad, E. Nassar, T. Roisnel, V. Dorcet, F. Chevallier, Z. Fajloun, F. Mongin, Synthesis 2017, 49, 4500-4516; d) N. M. Brikci-Nigassa, G. BentabedAbabsa, W. Erb, F. Chevallier, L. Picot, L. Vitek, A. Fleury, V. Thiery, M. Souab, T. Robert, S. Ruchaud, S. Bach, T. Roisnel, F. Mongin, Tetrahedron 2018, 74, 1785-1801.

[37] K. Rizwan, I. Karakaya, D. Heitz, M. Zubair, N. Rasool, G. A. Molander, Tetrahedron Lett. 2015, 56, 6839-6842.

[38] E. R. Strieter, B. Bhayana, S. L. Buchwald, J. Am. Chem. Soc. 2009, 131, 78-88.

[39] D. V. Kurandina, E. V. Eliseenkov, T. S. Khaibulova, A. A. Petrov, V. P. Boyarskii, Russ. J. Gen. Chem. 2015, 85, 2277-2281.
[40] H. E. Gottlieb, V. Kotlyar, A. Nudelman, J. Org. Chem. 1997, 62, 7512 7515

[41] A. Krasovskiy, P. Knochel, Synthesis 2006, 890-891.

[42] L. P. Hammett, G. H. Walden, Jr., S. M. Edmonds, J. Am. Chem. Soc 1934, 56, 1092-1094.

[43] A. I. Vogel, A. R. Tatchell, B. S. Furnis, A. J. Hannaford, P. W. G. Smith Vogel's textbook of practical organic chemistry, $5^{\text {th }}$ edn, Prentice Hall, 1996.

[44] S. Bugge, E. M. Skjoensfjell, F. B. Willumsen, E. Sundby, B. H. Hoff, Chem. Heterocycl. Compd. 2014, 50,1177-1187.

[45] a) J. M. L'Helgoual'ch, A. Seggio, F. Chevallier, M. Yonehara, E. Jeanneau, M. Uchiyama, F. Mongin, J. Org. Chem. 2008, 73, 177-183 b) M. Hedidi, G. Bentabed-Ababsa, A. Derdour, T. Roisnel, V. Dorcet, F. Chevallier, L. Picot, V. Thiéry, F. Mongin, Bioorg. Med. Chem. 2014, 22, 3498-3507.

[46] G. M. Sheldrick, Acta Crystallogr., Sect. A 2015, 71, 3-8.

[47] G. M. Sheldrick, Acta Crystallogr., Sect. C 2015, 71, 3-8.

[48] L. J. Farrugia, J. Appl. Crystallogr. 1997, 30, 565.

[49] A. Nemchik, V. Badescu, O. Phanstiel, Tetrahedron 2003, 59, 43154325.

[50] A. J. Biloski, B. Ganem, Synthesis 1983, 537-538.

[51] A. M. Berman, J. S. Johnson, J. Org. Chem. 2006, 71, 219-224.

[52] H. Shi, D. J. Babinski, T. Ritter, J. Am. Chem. Soc. 2015, 137, 37753778

[53] Z. Dong, G. Dong, J. Am. Chem. Soc. 2013, 135, 18350-18353.

[54] D. A. Shirley, M. D. Cameron, J. Am. Chem. Soc. 1952, 74, 664-665.

[55] K. R. Brower, E. D. Amstutz, J. Org. Chem. 1954, 19, 411-414.

[56] K.-J. Jung, S. B. Kang, J.-E. Won, S.-E. Park, K. H. Park, J. K. Park, S.G. Lee, Y.-J. Yoon, Synlett 2009, 490-494.

[57] Z. Lu, R. J. Twieg, Tetrahedron 2005, 61, 903-918.

[58] M. W. Hooper, M. Utsunomiya, J. F. Hartwig, J. Org. Chem. 2003, 68, 2861-2873.

[59] K. H. Hoi, S. Calimsiz, R. D. J. Froese, A. C. Hopkinson, M. G. Organ Chem. Eur. J. 2011, 17, 3086-3090.

[60] L. L. Joyce, R. A. Batey, Org. Lett. 2009, 11, 2792-2795.

[61] R. Uday Kumar, K. H. V. Reddy, B. S. P. Anil Kumar, G. Satish, V. P. Reddy, Y. V. D. Nageswar, Tetrahedron Lett. 2016, 57, 637-640.

[62] D. Cornelis, E. Franz, I. Asselberghs, K. Clays, T. Verbiest, G. Koeckelberghs, J. Am. Chem. Soc. 2011, 133, 1317-1327. 


\section{Entry for the Table of Contents}

Key topic: Thiophene aminations

\section{FULL PAPER}

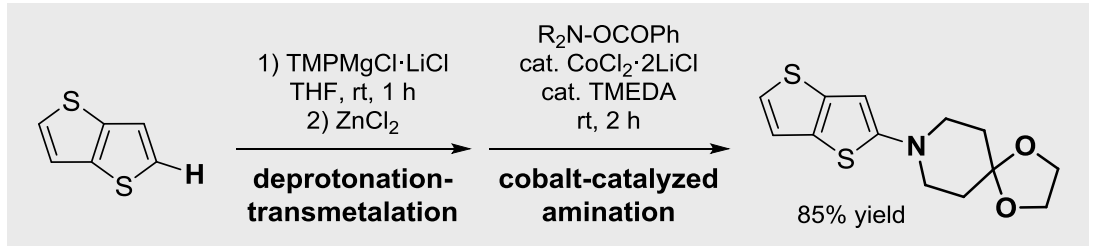

Both copper- and cobalt-catalyzed aminations of arylzincs using $\mathrm{N}$-benzoyloxy amines are possible. Thus, thienylzincs prepared by transmetalation from thienylmagnesium halides obtained by various methods including deprotometalation were aminated with success. In addition, triarylamines were prepared from aminothiophenes by consecutive copper-catalyzed $\mathrm{N}$-arylations using iodoarenes.
Salima Bouarfa, Simon Graßl, Maria Ivanova, Timothy Langlais, Ghenia Bentabed-Ababsa, * Frédéric Lassagne, William Erb, Thierry Roisnel, Vincent Dorcet, Paul Knochel* and Florence Mongin*

Page No. - Page No.

Copper- and Cobalt-Catalyzed Syntheses of Thiophene-Based Tertiary Amines 